\title{
Higgs, moduli problem, baryogenesis and large volume compactifications
}

\author{
Tetsutaro Higaki, ${ }^{a}$ Kohei Kamada, ${ }^{b}$ and Fuminobu Takahashi ${ }^{c}$ \\ ${ }^{a}$ Mathematical Physics Lab., RIKEN Nishina Center, \\ Saitama 351-0198, Japan \\ ${ }^{b}$ Deutsches Elektronen-Synchrotron DESY, \\ Notkestraße 85, D-22607 Hamburg, Germany \\ ${ }^{c}$ Department of Physics, Tohoku University, \\ Sendai 980-8578, Japan \\ E-mail: tetsutaro.higaki@riken.jp, kohei.kamada@desy.de, \\ fumi@tuhep.phys.tohoku.ac.jp
}

ABSTRACT: We consider the cosmological moduli problem in the context of high-scale supersymmetry breaking suggested by the recent discovery of the standard-model like Higgs boson. In order to solve the notorious moduli-induced gravitino problem, we focus on the LARGE volume scenario, in which the modulus decay into gravitinos can be kinematically forbidden. We then consider the Affleck-Dine mechanism with or without an enhanced coupling with the inflaton, taking account of possible Q-ball formation. We show that the baryon asymmetry of the present Universe can be generated by the Affleck-Dine mechanism in LARGE volume scenario, solving the moduli and gravitino problems. We also find that the overall volume modulus decays into a pair of the axionic superpartners, which contribute to the extra relativistic degrees of freedom.

Keywords: Flux compactifications, Supersymmetry Breaking, Cosmology of Theories beyond the SM, Supergravity Models

ARXIV EPRINT: 1207.2771 


\section{Contents}

1 Introduction 1

2 Affleck-Dine baryogenesis 2

2.1 Affleck-Dine baryogenesis in high-scale SUSY breaking 3

2.2 Effect of large negative Hubble induced mass 8

2.3 Discussion on the origin of the effective operator $K=|\phi|^{2}|I|^{2} / \tilde{M}^{2} \quad 9$

$\begin{array}{lll}\text { 2.3.1 Field-theoretical interpretation } & 10\end{array}$

3 LARGE volume scenario with three Kähler moduli in the geometric regime

$\begin{array}{lll}3.1 & \text { Moduli stabilization and its consequences } & 12\end{array}$

$\begin{array}{lll}3.1 .1 & \text { Moduli sector } & 12\end{array}$

$\begin{array}{lll}3.1 .2 & \text { Visible sector } & 15\end{array}$

3.1.3 A numerical example based on the model 2 in the literature [45] 16

$\begin{array}{ll}3.1 .4 \text { Higgs sector: } \mu / B \mu \text {-term } & 17\end{array}$

$\begin{array}{lll}3.2 & \text { Moduli problem in LVS } & 18\end{array}$

3.3 AD baryogenesis in LVS model in the geometric regime 21

4 LVS with three Kähler moduli in the singular regime 23

$\begin{array}{lll}5 & \text { Discussion and conclusions } & 27\end{array}$

$\begin{array}{ll}\text { A Example of } c & \mathbf{2 8}\end{array}$

A.1 Examples: heavy chiral fields 28

$\begin{array}{lll}\text { A.2 Examples: heavy vector field } & 29\end{array}$

\section{Introduction}

Recently the ATLAS and CMS collaborations have discovered a standard model (SM)-like Higgs particle with mass of about $125 \mathrm{GeV}$ [1] (see also [2, 3]). The relatively light Higgs boson mass strongly suggests the presence of new physics at a scale below the Planck scale [4]. From both phenomenological and theoretical points of view, supersymmetry (SUSY) is arguably the most plausible candidate for the new physics beyond the SM.

In the minimal supersymmetric extension of the SM, the $125 \mathrm{GeV}$ Higgs mass can be explained without invoking large stop mixing if the typical sparticle mass is at $O(10) \mathrm{TeV}$ or heavier. In particular, the SUSY should appear at a scale below $\mathrm{PeV}$ for $\tan \beta \gtrsim 2$, where $\tan \beta$ represents the ratio of the up-type and down-type Higgs boson vacuum expectation values (VEVs). It is therefore of utmost importance to study the cosmological and phenomenological implications of such high-scale SUSY suggested by the Higgs boson mass. 
The string theory is a plausible candidate for an underlying high-energy theory, and in particular, it seems to possess some features the quantum theory of gravity should possess. However, it suffers from a serious cosmological moduli problem [5-7]. Massless moduli fields parametrize the continuous ground state degeneracies and they generally appear in the compactifications of extra dimensions. In order to construct phenomenologically viable models, those moduli fields need to be stabilized. However, some of them remain relatively light, acquiring masses induced only by the SUSY breaking. Because of its light mass, those moduli are copiously produced after inflation as coherent oscillations. If the moduli mass is of order the weak scale or lighter, they typically decay after the big bang nucleosynthesis $(\mathrm{BBN})$, thus altering the standard cosmology in contradiction with observations.

In the case of high-scale SUSY as suggested from the SM-like Higgs boson mass, the moduli fields with mass heavier than $100 \mathrm{TeV}$ or so decay before the $\mathrm{BBN}$, and the cosmological moduli problem is greatly relaxed. However the moduli generically decay into gravitinos with a sizable branching fraction if kinematically allowed, and those gravitinos produce lightest SUSY particles (LSPs), whose abundance easily exceeds the observed dark matter density. This is known as the moduli-induced gravitino problem [8-11]. Furthermore, a huge amount of entropy is produced by the modulus decay, and any pre-existing baryon asymmetry would be diluted by a significant factor. Therefore it is difficult to generate the right amount of the baryon asymmetry via the standard leptogenesis [12], and we need more efficient baryogenesis such as the Affleck-Dine (AD) mechanism [13]. Thus, solving the issues of the moduli and gravitino problems and the origin of the baryon asymmetry is the key to understand the evolution of the Universe and high-energy theory.

Our strategy is twofold. First we revisit the AD mechanism in high-scale SUSY breaking, taking account of possible Q-ball formation [14-16]. We will show that the Q balls decay sufficiently fast and so they are cosmologically harmless, both because of the highscale SUSY breaking, and because of the mild hierarchy between the gaugino mass and the scalar mass. Secondly we consider the moduli problem in a realistic moduli stabilization. In order to solve the serious moduli-induced gravitino problem, we consider the LARGE volume scenario (LVS) [17], in which the modulus decay into gravitinos can be kinematically forbidden. Interestingly, the cut-off scale tends to be smaller than the Planck scale in LVS, and if the coupling between the inflaton and the AD field is enhanced, the resultant baryon asymmetry increases significantly. We will show that the right amount of the baryon asymmetry can be naturally generated by the AD mechanism in LVS, solving the moduli and gravitino problems.

The rest of the paper is organized as follows. In section 2 we consider the AD mechanism in high-scale SUSY breaking to study if it can generate a sufficient amount of the baryon asymmetry in the presence of a huge entropy production by the modulus decay. We give two concrete realizations of the moduli stabilization and discuss the cosmological issues in section 3 and section 4 . The last section is devoted for discussion and conclusions.

\section{Affleck-Dine baryogenesis}

We first review the $\mathrm{AD}$ mechanism $[13,18,19]$, in which the baryon asymmetry is generated thorough the dynamics of scalar fields with baryon and/or lepton charge. To be concrete 
we assume a mild hierarchy between gaugino and scalar masses, such that the scalar mass is several orders of magnitude heavier than the typical gaugino mass. Such mass spectrum is realized in simple anomaly mediation with a generic Kähler potential as well as in the modulus mediation, as we shall see in the next section.

We consider the effect of a large negative Hubble-induced mass on the AD mechanism and its cosmological consequences. Such an enhanced coupling of the AD field with the inflaton is expected in the context of LVS. We will see that the baryon asymmetry of the present Universe can be explained, even in the presence of a huge entropy production by the modulus decay. In particular, the Q balls decay before BBN, and so, they do not play any important role in our scenario.

\subsection{Affleck-Dine baryogenesis in high-scale SUSY breaking}

In supersymmetric theories, flat directions are ubiquitous [20, 21]. The scalar potential along flat directions vanishes in the exact SUSY limit at renormalizable level. A flat direction can be parameterized by a gauge invariant monomial such as $u d d$ or $L H_{u}$, and its dynamics can be described in terms of a complex scalar field $\phi$, which we call the AD field. If the $\mathrm{AD}$ field $\phi$ carries baryon and/or lepton charge, its dynamics can generate the baryon asymmetry in the Universe. Hereafter we assume that the AD field $\phi$ carries a non-vanishing baryon charge, $\beta$.

The flat direction is lifted by both non-renormalizable interactions and SUSY breaking. We consider a non-renormalizable superpotential of the following form,

$$
W=\frac{y}{M_{*}^{n-3}} \phi^{n},
$$

where $y$ is a coupling constant, $M_{*}$ is the cut-off scale, and $n$ is an integer greater than 3 . We set $y$ to be real and positive without loss of generality. The value of $n$ depends on flat directions as well as on the existence of a possible discrete symmetry under which $\phi$ is charged. Taking account of soft SUSY-breaking effects, the scalar potential in a flat space time is expressed as,

$$
V(\phi)=m_{0}^{2}|\phi|^{2}+\left(\frac{A_{n} y}{M_{*}^{n-3}} \phi^{n}+\text { h.c. }\right)+\frac{n^{2} y^{2}}{M_{*}^{2 n-6}}|\phi|^{2 n-2},
$$

where $m_{0}$ and $A_{n}$ are the soft scalar mass of $\phi$ and the coefficient of the $A$-term, respectively. The magnitude of $m_{0}$ and $A_{n}$ depend on the SUSY-breaking mediation mechanism. The $A$-term violates the baryon number explicitly, which is the source of baryon asymmetry. Hereafter we assume that $m_{0}$ is of order $100 \mathrm{TeV}$ or heavier, in order to explain the SM-like Higgs boson with mass about $125 \mathrm{GeV}$. As a reference value we will set $m_{0}=10^{3} \mathrm{TeV}$ in the following analysis.

Now we consider the dynamics of the AD field in the inflationary Universe. In the case where inflation is driven by the $F$-term of a canonically normalized inflaton, $I$, the AD field generally acquires a mass squared whose amplitude is of order the Hubble parameter squared, through the Planck-suppressed interaction in supergravity. We here assume that 
the sign of the Hubble-induced mass is negative, ${ }^{1}$

$$
V_{H}=-c^{2} H^{2}|\phi|^{2}
$$

where $H$ is the Hubble parameter and $c$ is a positive numerical coefficient. For the moment, we consider the case of $c=O(1)$. Such a negative mass term is generated if there is a quartic coupling between inflaton and the AD field in the Kähler potential,

$$
K_{\mathrm{NM}}=\frac{a}{M_{\mathrm{pl}}}|\phi|^{2}|I|^{2},
$$

where $a$ is a positive numerical coefficient of order unity and $M_{\mathrm{pl}}$ is the reduced Planck mass.

Suppose that the Hubble-induced mass $c H$ during inflation is larger than the soft scalar mass $m_{0}$. Then, the origin of $\phi$ is destabilized and it settles down at the potential minimum determined by the balance between the negative Hubble-induced mass and the $F$-term from the non-renormalizable superpotential, acquiring a large expectation value:

$$
\left|\phi_{\mathrm{inf}}\right| \simeq\left(\frac{c H_{\mathrm{inf}} M_{*}^{n-3}}{n \sqrt{n-1} y}\right)^{1 /(n-2)},
$$

where $H_{\text {inf }}$ is the Hubble parameter during inflation. Such initial condition is one of the requisites for the $\mathrm{AD}$ mechanism, namely,

$$
H_{\mathrm{inf}}>\frac{m_{0}}{c},
$$

is required.

Let us study the dynamics of the AD field $\phi$ after inflation and see how the baryon asymmetry is generated. In the single-field inflation, $\phi$ still receives a negative Hubbleinduced mass after inflation, as long as the energy density of the Universe is dominated by the inflaton matter. In the case of multi-field inflation, we assume that the Hubble-induced mass term is negative and its magnitude does not change significantly during and after inflation. After inflation, $\phi$ then follows the time-dependent minimum $\left(c H M_{*}^{n-3} / n \sqrt{n-1} y\right)^{1 /(n-2)}$ until $\phi$ starts oscillations around the origin when $H \simeq m_{0} / c .^{2}$ At the onset of oscillations, $\phi$ is kicked into the phase direction by the $A$-term, and the baryon number is generated. The resultant baryon number density,

$$
n_{B}=i \beta\left(\phi \dot{\phi}^{*}-\dot{\phi} \phi^{*}\right)
$$

can be evaluated by solving the equation of evolution,

$$
\dot{n}_{B}+3 H n_{B}=2 \beta \operatorname{Im}\left(\frac{\partial V}{\partial \phi} \phi\right)=\frac{2 n \beta A_{n} y}{M_{*}^{n-3}} \operatorname{Im}\left(\phi^{n}\right) .
$$

\footnotetext{
${ }^{1}$ If the sign of the Hubble induced mass is positive, the AD field settles down to the origin during inflation and it does not play any important role in cosmology.

${ }^{2}$ Here we assume that the thermal correction to the scalar potential does not affect the dynamics of $\phi$. This is considered to be the case if the field value of $\phi$ is sufficiently large.
} 
Assuming that the scale factor evolves as $a \propto t^{2 / 3}$ during inflaton matter domination, we obtain the resultant baryon number density at $t>t_{\mathrm{osc}}$ :

$$
\begin{aligned}
n_{B}(t) & =\frac{2 \beta}{a^{3}(t)} \int^{t} d t^{\prime} a^{3}\left(t^{\prime}\right) \operatorname{Im}\left(\frac{\partial V}{\partial \phi} \phi\right) \\
& \simeq\left(\frac{a\left(t_{\mathrm{osc}}\right)}{a(t)}\right)^{3} \frac{2(n-2)}{3 n^{2 /(n-2)}(n-1)^{n /(2 n-4)}(n-3)} \frac{\beta c^{n /(n-2)}}{y^{2 /(n-2)}} A_{n} M_{*}^{2(n-3) /(n-2)} H_{\mathrm{osc}}^{2 /(n-2)} \delta_{\mathrm{eff}} \\
& \simeq\left(\frac{a\left(t_{\mathrm{osc}}\right)}{a(t)}\right)^{3} \frac{2(n-2)}{3 \sqrt{n-1}(n-3)} \beta c A_{n} \phi_{\mathrm{osc}}^{2} \delta_{\mathrm{eff}},
\end{aligned}
$$

where the subindex 'osc' means that the variable is evaluated at the onset of the oscillations. Here $\delta_{\text {eff }} \leq 1$ is the CP phase factor, and it is typically of $\mathcal{O}(0.1)$, without fine-tunings of the initial phase of the $\mathrm{AD}$ field. The baryon asymmetry in a comoving volume $a^{3} n_{B}$ is conserved soon after the onset of the AD field oscillation, because the oscillation amplitude of $\phi$ decreases rapidly due to the Hubble friction.

So far we have assumed that the oscillation in the phase direction does not begin until the onset of the oscillation in the radial direction. This assumption is valid as long as the $A$-term is small enough,

$$
\left|A_{n}\right|<\frac{\sqrt{n-1}}{n^{2} c^{2}} m_{0} \equiv A_{n}^{\text {cri }}
$$

If the $\mathrm{AD}$ field starts to oscillate in the phase direction earlier, the estimate of the resultant baryon asymmetry becomes a little more complicated. Dividing the AD field into the radial and phase component, $\phi=|\phi| e^{i \theta}$, the equation of motion for the phase component reads,

$$
\ddot{\theta}+\left(3 H+2 \frac{|\dot{\phi}|}{|\phi|}\right) \dot{\theta}+\frac{A_{n} y}{M_{*}^{n-3}}|\phi|^{n-2} \sin (n \theta)=0 .
$$

Substituting the expression $|\phi| \sim\left(c H M_{*}^{n-3} / \sqrt{n-1} y\right)^{1 /(n-2)}$, this equation can be approximated as

$$
\ddot{\theta}+\frac{2(n-3)}{(n-2) t} \dot{\theta}+\frac{2 n c A_{n}}{3 \sqrt{n-1} t} \theta=0 .
$$

Here we have assumed that $\theta$ is not so large that one can approximate $\sin (n \theta) \sim n \theta$ and used $H=2 / 3 t$. Solving eq. (2.12) for $n=6$, we obtain an approximate solution after the onset of the oscillation along the phase direction,

$$
\theta(t) \simeq \sqrt{\frac{t_{\mathrm{ph}}}{t}} \cos \left(2 \sqrt{\frac{t}{t_{\mathrm{ph}}}}\right) \theta_{0},
$$

where $t_{\mathrm{ph}}=\left(2 n c A_{n} / 3 \sqrt{n-1}\right)^{-1}$ is the time at the onset of the oscillation in the phase direction and $\theta_{0}$ is the $\mathrm{AD}$ field phase at $t=t_{\mathrm{ph}}$. Therefore, the time derivative of $\theta$ at the onset of its oscillation along the radial direction is estimated as

$$
\dot{\theta}\left(t_{\mathrm{osc}}\right) \sim \frac{m_{0}}{c} \delta_{\mathrm{eff}},
$$


where we include the overall CP phase factor $\delta_{\text {eff }}=\theta_{0} \delta_{\text {osc }}$. Here $\delta_{\text {osc }}$ represents how efficient the phase velocity turns into the baryon asymmetry at $t=t_{\mathrm{osc}}$. Then, we can approximate the baryon number density at the onset of the AD field oscillation in the radial direction,

$$
n_{B}\left(t_{\mathrm{osc}}\right) \simeq \beta \phi_{\mathrm{osc}}^{2} \dot{\theta} \sim \frac{m_{0}}{c} \phi_{\mathrm{osc}}^{2} \delta_{\mathrm{eff}} .
$$

Similar to the former case, the baryon asymmetry in the comoving volume is fixed soon after the onset of the $\mathrm{AD}$ field oscillation in the radial direction. As a result, we arrive at an approximate estimation,

$$
n_{B}(t) \simeq\left(\frac{a\left(t_{\mathrm{osc}}\right)}{a(t)}\right)^{3} \beta c A_{n} \phi_{\mathrm{osc}}^{2} \delta_{\mathrm{eff}} \times f
$$

with

$$
f \equiv \begin{cases}1 \quad \text { for } & A_{n}<A_{n}^{\text {cri }}, \\ \frac{A_{n}^{\text {cri }}}{A_{n}} \text { for } & A_{n}>A_{n}^{\text {cri }},\end{cases}
$$

where we have omitted an $\mathcal{O}(1)$ numerical factor. Note that there is an upper bound on $c$,

$$
c<\frac{\sqrt{n-1} H_{\mathrm{inf}}}{n A_{n}},
$$

in order for the $\mathrm{AD}$ field not to settle down to the potential minimum in the phase direction during inflation. Moreover, for the case $c \sim \mathcal{O}(1), A_{n}$ should not be much larger than $A_{n}^{\text {cri }}$ since there arises a color-breaking potential minimum other than the origin in the scalar potential.

The present baryon-to-entropy ratio is then evaluated as

$$
\begin{aligned}
\frac{n_{B}}{s}\left(t_{0}\right) & \simeq \Delta^{-1} \frac{n_{B}}{s}\left(t_{R}\right) \\
& \simeq \frac{n(n-2)}{6 \sqrt{n-1}(n-3)} \beta c^{3} f \delta_{\mathrm{eff}} \Delta^{-1} \frac{A_{n} T_{R}}{m_{0}^{2}}\left(\frac{\phi_{\mathrm{osc}}}{M_{\mathrm{pl}}}\right)^{2} \\
& \sim 10^{-10} c^{3} f \delta_{\mathrm{eff}}\left(\frac{\Delta^{-1}}{10^{-3}}\right)\left(\frac{A_{n}}{10^{3} \mathrm{GeV}}\right)\left(\frac{T_{R}}{10^{7} \mathrm{GeV}}\right)\left(\frac{m_{0}}{10^{6} \mathrm{GeV}}\right)^{-2}\left(\frac{\phi_{\mathrm{osc}}}{10^{16} \mathrm{GeV}}\right)^{2},
\end{aligned}
$$

where $T_{R}$ is the reheating temperature. Here we have inserted the entropy dilution factor $\Delta$ due to the modulus decay. Note that if there is a moduli dominated era, the baryon asymmetry is diluted by a factor $\Delta^{-1} \simeq T_{X} / T_{\text {dom }}$ where the subindices $X$ and 'dom' represent that the variables are evaluated at the modulus decay and at the onset of the moduli domination, respectively.

Now we turn to the cosmological effect of Q balls [14-16], which are potential obstacles in this scenario. A Q ball is a non-topological soliton, and the Q-ball solution exists when the scalar potential is flatter than the quadratic potential, and its stability is guaranteed by the conserved (baryon and/or lepton) charge. In the AD mechanism, this condition on the scalar potential is met if the radiative correction to the soft mass is negative $[15,16$, $22,23]$, i.e.,

$$
V(\phi) \ni m_{0}^{2}|\phi|^{2}\left(1+K \log \left(\frac{|\phi|^{2}}{\Lambda^{2}}\right)\right)
$$


with $K<0$. Here $\Lambda$ is the SUSY-breaking scale where $m_{0}$ is evaluated. $K$ is a numerical coefficient of the one-loop radiative corrections and it becomes negative when the gaugino loop dominates. For example, in case of the $u d d$ flat direction, $K$ is evaluated as $[22,23]$

$$
K \simeq-\frac{4 \alpha_{3}}{3 \pi} \frac{M_{3}^{2}}{m_{0}^{2}} \simeq 10^{-6} \times\left(\frac{M_{3}}{10^{4} \mathrm{GeV}}\right)^{2}\left(\frac{m_{0}}{10^{6} \mathrm{GeV}}\right)^{-2}
$$

where we have considered only gluino contributions. Note that we consider a case where there is a hierarchy between the scalar mass $m_{0}$ and the gaugino mass $M_{a}$. For example, in the anomaly mediation with a general Kähler potential, the hierarchy is given by $M_{a} / m_{0} \sim$ $M_{a} / m_{3 / 2} \sim g_{a}^{2} / 16 \pi^{2}=10^{-3}-10^{-2}$. As a result, $|K|$ is suppressed compared to the case of $m_{0} \simeq M_{a}{ }^{3}$

If $K$ is negative, the AD field condensate experiences spatial instabilities and Q balls are formed at about $H=H_{*} \sim 0.1 m_{0}|K|$. The Q balls have the following properties [22-25],

$$
R \simeq|K|^{-1 / 2} m_{0}^{-1}, \quad \omega \simeq m_{0}, \quad \phi_{Q} \simeq \frac{H_{*}}{H_{\mathrm{osc}}} \phi_{\mathrm{osc}} \simeq 0.1 c|K| \phi_{\mathrm{osc}}
$$

where $R$ is the radius of a $\mathrm{Q}$ ball, $\omega$ is the angular momentum of the AD field inside a $\mathrm{Q}$ ball, and $\phi_{Q}$ is the field value at the center of a $\mathrm{Q}$ ball. The charge and the energy stored in a $\mathrm{Q}$ ball are estimated as

$$
Q \simeq R^{3} \omega \phi_{Q}^{2} \simeq 10^{15} c^{2}\left(\frac{|K|}{10^{-6}}\right)^{1 / 2}\left(\frac{m_{0}}{10^{6} \mathrm{GeV}}\right)^{-2}\left(\frac{\phi_{\text {osc }}}{10^{16} \mathrm{GeV}}\right)^{2}, \quad E_{Q} \simeq m_{0} Q .
$$

If the Q balls are stable, they may overclose the Universe. Alternatively, if they are unstable and decay into lighter degrees of freedom during BBN, it may change the light element abundances spoiling the success of the BBN. In the present case, however, the energy per unit charge, $E / Q$, is comparable to the soft scalar mass, $m_{0}$, and hence they can decay into quarks and lighter SUSY particles. The decay proceeds from the Q-ball surface and its rate is evaluated as [26]

$$
\begin{aligned}
\Gamma_{Q} & =\frac{1}{Q} \frac{d Q}{d t} \simeq \frac{\omega^{3} R^{2}}{48 \pi Q} \\
& \simeq 10^{-5} \mathrm{GeV}^{-2}\left(\frac{|K|}{10^{-6}}\right)^{-3 / 2}\left(\frac{m_{0}}{10^{6} \mathrm{GeV}}\right)^{3}\left(\frac{\phi_{\mathrm{osc}}}{10^{16} \mathrm{GeV}}\right)^{-2} .
\end{aligned}
$$

Thus, the decay temperature of the $\mathrm{Q}$ balls is

$$
T_{\mathrm{dec}} \simeq \sqrt{\Gamma_{Q} M_{\mathrm{pl}}} \simeq 5 \times 10^{6} \mathrm{GeV}^{-1}\left(\frac{|K|}{10^{-6}}\right)^{-3 / 4}\left(\frac{m_{0}}{10^{6} \mathrm{GeV}}\right)^{3 / 2}\left(\frac{\phi_{\mathrm{osc}}}{10^{16} \mathrm{GeV}}\right)^{-1} .
$$

Therefore, the Q balls decay much before BBN even if $\phi_{\text {osc }}$ is as large as the Planck scale, both because of the heavy scalar mass and because of the small value of $|K|$. The $\mathrm{Q}$ balls are cosmologically harmless in the high-scale SUSY breaking.

\footnotetext{
${ }^{3}$ It is possible that top (and bottom) loop contributions make $K$ positive, depending on the value of tan $\beta$. In this case $\mathrm{Q}$ balls are not formed.
} 


\subsection{Effect of large negative Hubble induced mass}

So far, we have assumed that the negative mass of the AD field during and after inflation is of order the Hubble parameter, i.e., $c \simeq \mathcal{O}(1)$. Here let us consider the case where the coupling of the inflaton and the AD field in the Kähler potential is enhanced,

$$
K_{\mathrm{NM} 2}=\frac{1}{\tilde{M}^{2}}|\phi|^{2}|I|^{2}, \quad \tilde{M} \ll M_{\mathrm{pl}} .
$$

Then the large negative Hubble-induced mass is generated,

$$
V_{\mathrm{H}}=-c^{2} H^{2}|\phi|^{2}, \quad c \simeq \frac{M_{\mathrm{pl}}}{\tilde{M}},
$$

where $\tilde{M}$ denotes the effective cut-off scale for the enhanced coupling. Such an enhancement is indeed realized in the string inspired models as we shall see later.

The large negative Hubble induced mass delays the onset of the AD field oscillation. Then, the Hubble friction is suppressed when the AD field starts to oscillate. One may worry that, since the AD field oscillates many times in one Hubble time, the AD field dynamics after the onset of its oscillations may wash out the baryon asymmetry generated by the first kick and the resultant baryon asymmetry may be suppressed. However, because the time scale of the first kick, $H_{\text {osc }}^{-1} \simeq \mathrm{cm}_{0}^{-1}$, is much longer than the typical time scale of oscillations, $m_{0}^{-1}$, the effect of the wash out tends to be negligibly small. As a result, the baryon asymmetry is determined by the first kick and becomes fixed soon after the commencement of the oscillations. The baryon asymmetry is therefore given by

$$
\frac{n_{B}}{s}\left(t_{0}\right) \sim 10^{-10} f \delta_{\text {eff }}\left(\frac{c}{10}\right)^{3}\left(\frac{\Delta^{-1}}{10^{-3}}\right)\left(\frac{A_{n}}{10^{3} \mathrm{GeV}}\right)\left(\frac{T_{R}}{10^{6} \mathrm{GeV}}\right)\left(\frac{m_{0}}{10^{6} \mathrm{GeV}}\right)^{-2}\left(\frac{\phi_{\mathrm{osc}}}{10^{15} \mathrm{GeV}}\right)^{2} .
$$

Note that the resultant baryon-to-entropy-ratio is enhanced by a factor of $c^{3}$ or $c$. (Note that $f$ depends on $c$ if the A-term is large.)

The later onset of the AD field oscillation also affects the Q-ball properties. This results in the larger field value of the $\mathrm{AD}$ field at the center of $\mathrm{Q}$ balls and the larger charge stored in a $\mathrm{Q}$ ball. Although the large charge of $\mathrm{Q}$ balls suppresses the decay rate by a factor of $c^{-2}$ (eq. (2.26)), the Q balls can decay before BBN for a wide range of $c$. However, in order to avoid the overproduction of the LSP produced by the Q-ball decay, $c$ should not be too large. Typically it should satisfy $c \lesssim 10^{3}$. The precise value of the upper bound depends on the thermal history of the Universe. If the modulus field decays later, the LSP produced by the Q-ball decay is diluted. However, as shown in ref. [8, 9], the moduli tends to have a sizable (not chiral suppressed) branching fraction of the decay into gauginos. So in this case the LSPs may be still overproduced. That said, as we shall see later, the modulus field decays sufficiently fast in the concrete moduli stabilization because of the heavy gravitino mass. Therefore, the upper bound on $c \lesssim 10^{3}$ is a more or less reasonable constraint in our scenario.

In summary, the large negative Hubble induced mass enhances the baryon asymmetry of the Universe. This will help the situation of string-inspired SUSY-breaking models where the baryogenesis is difficult due to the late time entropy production from modulus decay. 
Furthermore, the inflation scale is generically bounded above in order not destabilize the moduli fields. It is therefore non-trivial if the AD mechanism works in such low-scale inflation model, because the AD field may be stabilized at the origin during inflation. However, if $c \gg 1$, the origin can be destabilized by the large negative Hubble-induced mass term, the $\mathrm{AD}$ mechanism becomes viable.

\subsection{Discussion on the origin of the effective operator $K=|\phi|^{2}|I|^{2} / \tilde{M}^{2}$}

Before studying the moduli stabilization, let us discuss various possibilities of the origin of the effective operator in eq. (2.28). The structure of this term strongly depends on what the inflaton $I$ is, e.g. its Kähler potential [27-29]. For instance, the volume modulus inflation [30] and the warped D-brane inflation [31] are not suitable. In this paper, instead, we treat the inflaton as the usual chiral matter-like fields including open string moduli on the visible brane for reheating the visible sector: $K_{\text {inflaton }} \sim|I|^{2}$.

When a massive mode is propagating between the inflaton and the AD field, the cutoff $\tilde{M}$ in the Kähler potential (2.28) is given by the mass after integrating it out. In the string theories, there are many mass scales such as Kaluza-Klein (KK) scale, the string scale and winding scale. With the $6 \mathrm{D}$ compactification volume of extra dimension $\mathcal{V} \equiv R^{6} M_{\text {string }}^{6}$, they are written as ${ }^{4}$

$$
M_{K K} \sim \frac{1}{R} \sim \frac{M_{\mathrm{pl}}}{\mathcal{V}^{2 / 3}}, \quad M_{\text {string }} \sim \frac{M_{\mathrm{pl}}}{\mathcal{V}^{1 / 2}}, \quad M_{\text {wind }} \sim M_{\mathrm{string}}^{2} R \sim \frac{M_{\mathrm{pl}}}{\mathcal{V}^{1 / 3}}
$$

in the large volume limit. Here we ignored the string coupling dependence. Note that the above KK and winding modes are the ones propagating in the bulk while the local KK and winding modes relevant to the local models are estimated as $M_{K K}^{\prime} \sim M_{\mathrm{pl}} /\left(\mathcal{V}^{1 / 2} \tau^{1 / 4}\right)$ and $M_{\text {wind }}^{\prime} \sim M_{\mathrm{pl}} \tau^{1 / 4} / \mathcal{V}^{1 / 2}$ respectively, where $\tau$ is a local modulus describing a 4-cycle. There will be also massive modes in the Landau level on the magnetized branes and its T-dual modes among intersecting D-branes, and their masses are given by of order $M_{K K}$ ( or $M_{K K}^{\prime}$ ) and of order $M_{\text {string }}$, respectively. If such massive modes are coupled to the light modes of our interest, the operator in eq. (2.28), where $\tilde{M}$ is identified with $M_{K K}, M_{\text {string }}, M_{\text {wind }}$ etc., will appear after integrating them out [32-37]. Then the enhancement factor of the Hubble-induced mass term reads

$$
c \sim \mathcal{V}^{2 / 3}, \quad \mathcal{V}^{1 / 2} \quad \text { or } \quad \mathcal{V}^{1 / 3}
$$

with the canonically normalized fields. Thus it is possible to realize

$$
c \gg 1
$$

in the large $\mathcal{V}$ limit. Note that the condition (2.10) is not satisfied for the above values of $c$, and so, the phase of the AD field starts to oscillate before the radial component.

\footnotetext{
${ }^{4}$ Here the string scale comes from the coefficient in front of the $10 \mathrm{D}$ Einstein term: $M_{\text {string }}^{8} \int *_{10} \mathcal{R} \rightarrow$ $M_{\text {string }}^{2} \mathcal{V} \int *_{4} \mathcal{R} \equiv M_{\mathrm{pl}}^{2} \int *_{4} \mathcal{R}$ up to the string coupling. The winding modes here are open strings which are wrapping on a cycle in the extra dimension or which are stretching between two branes.
} 


\subsubsection{Field-theoretical interpretation}

Here let us interpret the behavior discussed above from the view point of the field theory. We emphasize again that after integrating out massive mode propagating between the light modes, the cutoff $\tilde{M}$ is given by the mass of the massive mode. The low-energy effective theories must be such that the cutoff $\tilde{M}$ corresponds to the scale of the massive mode.

Consider first the effective theory with heavy chiral multiplets $\left(\Phi_{H}+\Phi_{H}^{c}\right):^{5}$

$$
\begin{aligned}
K & =Z_{H}\left(\left|\Phi_{H}\right|^{2}+\left|\Phi_{H}^{c}\right|^{2}\right)+Z_{I}|I|^{2}+Z_{\phi}|\phi|^{2}, \\
W_{\text {heavy }} & =M_{H} \Phi_{H} \Phi_{H}^{c}+\Phi_{H}^{c} \phi I .
\end{aligned}
$$

Here $M_{H}=M_{\mathrm{pl}}$ is expected from the holomorphicity ${ }^{6}$ and $Z$ is the Kähler metric that depends on closed string moduli. After integrating out $\left(\Phi_{H}+\Phi_{H}^{c}\right)$ and the canonical normalizing $I_{c}=Z_{I}^{1 / 2} I$ and $\phi_{c}=Z_{\phi}^{1 / 2} \phi$, we obtain the effective operator,

$$
K_{\text {low }}=\frac{\left|I_{c}\right|^{2}\left|\phi_{c}\right|^{2}}{\tilde{M}^{2}}, \quad \text { with } \tilde{M}^{2}=\frac{Z_{\phi} Z_{I}}{Z_{H}} M_{\mathrm{pl}}^{2}
$$

The $\tilde{M}$ in string theories should correspond to the mass scale discussed above, even though it is difficult to obtain the information of the relevant wavefunction $Z$.

In global string models sitting on the bulk, $\tilde{M}$ will be of order the Kaluza-Klein scale. In local models, it will be around the string scale for the cancelled RR-tadpole case or a winding mode scale for the (global) uncancelled RR-tadpole case [38-41]. Those are because there are no Kaluza-Klein modes on the local brane and for the latter case the winding modes are necessary to cancel the global RR-tadpole.

Next, let us consider the case with a heavy $\mathrm{U}(1)$ vector multiplet $V_{H}$ :

$$
K=\left|I_{c}\right|^{2} e^{2 g q_{I} V_{H}}+\left|\phi_{c}\right|^{2} e^{2 g q_{\phi} V_{H}}+\frac{1}{2} M_{H}^{2} V_{H}^{2} .
$$

Here $g$ is the $\mathrm{U}(1)$ coupling, $q_{I}$ and $q_{\phi}$ are the $\mathrm{U}(1)$ charges of $I$ and $\phi$. Since we have assumed that inflaton $I$ is a matter-like field, it is plausible for $I$ to have the $\mathrm{U}(1)$ charge. Let us consider the origin of the mass term in two ways. In string theories, $V_{H}$ is typically a (non-)anomalous $\mathrm{U}(1)$ vector multiplet and hence the mass term $M_{H}$ comes from the Stückelberg coupling between the U(1) and string moduli $\Phi$

$$
K_{\text {moduli }}=K\left(\Phi+\Phi^{\dagger}+V_{H}\right) \supset K^{\prime} V+\frac{1}{2} K^{\prime \prime} V_{H}^{2} \equiv \xi_{\mathrm{FI}} V+\frac{1}{2 g^{2}} M_{H}^{2} V_{H}^{2} .
$$

Then $M_{H}$ is given by the Kähler metric of the relevant moduli in the vanishing FayetIliopoulos (FI) term limit, $\xi_{\mathrm{FI}}=0$. On the other hand, if the above FI term is non-zero, i.e. $K^{\prime} \neq 0$, a canonically normalized matter-like field $\psi_{c}$ charged under the $\mathrm{U}(1)$ symmetry

\footnotetext{
${ }^{5}$ On the flat space in the extra dimension, $\Phi_{H}$ can not be KK mode because the momentum conservation is violated while massive modes in the Landau level on the magnetized brane (and its T-dual) would be viable even in that case.

${ }^{6}$ If $\Phi_{H} \Phi_{H}^{c}$ is charged under an anomalous U(1) symmetry, $M_{H}$ will be a function of the moduli; it is possible to obtain $M_{H} \ll M_{\mathrm{pl}}$ at the non-perturbative level. We ignored possible term of $K \supset \Phi_{H}^{\dagger} I \phi$ for a simplicity.
} 
will condense to cancel it via the D-term condition, $D \sim K^{\prime}+q_{\psi}\left|\psi_{c}\right|^{2} \sim 0$. Then one will find the mass term of $V_{H}$ :

$$
K_{\psi}=\left|\psi_{c}\right|^{2} e^{2 g q_{\psi} V_{H}} \quad \rightarrow \quad M_{H}^{2} \sim g^{2}\left\langle\left|\psi_{c}\right|^{2}\right\rangle \sim g^{2}\left|K^{\prime}\right|,
$$

for $\left|K^{\prime}\right| \gg K^{\prime \prime}$. After integrating out $V_{H}$ via $\partial_{V_{H}} K=0$ in eq. (2.37) up to the kinetic term of the gauge field, one finds

$$
\begin{aligned}
K_{\text {low }} & =-4 g^{2} q_{I} q_{\phi} \frac{\left|I_{c}\right|^{2}\left|\phi_{c}\right|^{2}}{M_{H}^{2}} \\
\tilde{M}^{2} & =\frac{M_{H}^{2}}{4 g^{2}\left|q_{\phi} q_{I}\right|}=\frac{K^{\prime \prime}}{4\left|q_{\phi} q_{I}\right|} \quad \text { or } \quad \frac{\left|K^{\prime}\right|}{4\left|q_{\phi} q_{I}\right|} \quad \text { for } q_{I} q_{\phi}<0 .
\end{aligned}
$$

Note that the Goldstone multiplet eaten by $V_{H}$ is a modulus $\Phi$ in the former case, whereas it is $\psi_{c}$ in the latter case. Here $M_{H}$ will be of order string scale for anomalous U(1) case, whereas for the case of non-anomalous massive multiplet [42] it will be less than of order the Kaluza-Klein scale and the relevant gauge coupling will depend on compactification volume. This is because the non-anomalous U(1) multiplet becomes massive due to the anomaly in the compact extra dimension. We also provide a few examples in the appendix.

\section{LARGE volume scenario with three Kähler moduli in the geometric regime}

In this section, we shall study a supergravity model inspired by the string theory, in which there are lots of string moduli through the compactification. In a string model compactified on a Calabi-Yau space, there will be the dilaton $S$ determining the string coupling, the Kähler (volume) moduli $T$ and the complex structure (shape) ones $U$. They should be stabilized because an ultralight moduli can mediate fifth forth among matter fields and the moduli determine the size of not only compactification but also of physical parameters such as a gauge coupling. Therefore moduli stabilization is mandatory in string theories. For it, closed string flux backgrounds in extra dimensions, i.e. flux compactifications, are powerful tools to fix a lot of the moduli simultaneously. The remaining moduli which is not stabilized by the fluxes can become massive by instantons/gaugino condensation. By combination of them, all the moduli can be stabilized on a Calabi-Yau space [43]. In the followings, we will use the language in the type IIB orientifold flux compactification on the Calabi-Yau space [44].

On the other hand, the origin of the low cutoff scale $\tilde{M}$ in eq. (2.28) will be naturally explained in terms of large volume compactification. However, such moduli relevant to the large volume typically has a long lifetime and hence may cause cosmological disasters, diluting any pre-existing baryon asymmetry. In the following subsections, the cutoff scale and the dilution factor will be estimated. The supergravity computations will be done in the Planck unit $\left(M_{\mathrm{pl}}=1\right)$, whereas the Planck scale will be shown explicitly in the physical quantities. 


\subsection{Moduli stabilization and its consequences}

\subsubsection{Moduli sector}

From this subsection, let us study an explicit supergravity model, which is so-called LARGE volume scenario (LVS) [17] studied in [45]. (See also [46] and [47].) In such a scenario, a swiss-cheese type Calabi-Yau geometry and a negative Euler number on it ${ }^{7}$ will be required.

In this type IIB orientifold model, the dilaton and the complex structures will be stabilized by the fluxes while the Kähler moduli will be fixed by the non-perturbative effect. We will focus on the Kähler moduli $T$ relevant to the low energy physics and then mention the expected consequences of the dilaton and the complex structure moduli. The effective theory of Kähler moduli $T_{i}(i=1,2,3)$ are given by

$$
\begin{aligned}
K & =-2 \log \left(\mathcal{V}+\frac{\xi}{2}\right), \\
\mathcal{V} & =\left(\eta_{1} \tau_{1}\right)^{3 / 2}-\left(\eta_{2} \tau_{2}\right)^{3 / 2}-\left(\eta_{3} \tau_{3}\right)^{3 / 2}, \quad \xi=-\frac{\chi \zeta(3)}{2(2 \pi)^{3} g_{s}^{3 / 2}}, \\
W & =A e^{-a\left(T_{2}+C_{1} e^{-2 \pi T_{3}}\right)}-B e^{-b\left(T_{2}+C_{2} e^{-2 \pi T_{3}}\right)}, \\
& =A e^{-a T_{2}}-B e^{-b T_{2}}-\left(a A C_{1} e^{-a T_{2}}-b B C_{2} e^{-b T_{2}}\right) e^{-2 \pi T_{3}}+\cdots, \\
& \equiv W_{\text {eff }}+A_{\text {eff }} e^{-2 \pi T_{3}}+\cdots .
\end{aligned}
$$

In the followings, we will take

$$
\begin{gathered}
\eta_{i=1,2,3}=\mathcal{O}(0.1-1), \quad g_{s}=\mathcal{O}(0.1), \quad C_{i=1,2}, a, b=\mathcal{O}(1), \quad \chi=-\mathcal{O}(100)<0 \\
A, B \gg 1
\end{gathered}
$$

Although choosing $A, B \gg 1,\left\langle W_{\text {eff }}\right\rangle \ll 1$ will be obtained naturally due to the exponentially suppressed racetrack stabilization. Such large parameters may be explained through fluxed branes. We decomposed moduli fields as

$$
T_{i}=\tau_{i}+i \sigma_{i}
$$

It is easy to note that $\tau_{1}$ becomes the lightest modulus and $\sigma_{1}$ is massless because the former is not included in the superpotential and the latter is absent from the whole potential. Here $g_{s}$ is the string coupling fixed via flux compactification and $\chi$ is the Euler number of the Calabi-Yau space: $\chi=2\left(h^{11}-h^{21}\right)=2\left(3-h^{21}\right)<0$. (Here we assumed $h_{-}^{11}=h_{+}^{21}=0$.) $\tau_{1}$ originates from the bulk volume 4-cycle of the extra dimension while $\tau_{2}$ and $\tau_{3}$ come from the local 4-cycle volume. Their axionic partners $\sigma_{i}$ do from RR 4-form on the relevant 4cycles. Three $\eta_{i}$ are related with the intersection number in the Calabi-Yau space between three 2-cycles $\sim \tau_{i}^{1 / 2}$. The superpotential comes from the double gaugino condensations on the separate two stacks of D7-branes wrapping on the rigid 4-cycle (divisor) ${ }^{8}$ whose

\footnotetext{
${ }^{7}$ With a singular cycle and a proper quantum effect from the large volume cycle, a positive Euler number will be also available for LVS [48].

${ }^{8}$ Here, on the D-brane wrapping on the rigid cycle, there are no light adjoint matter fields called open string moduli which are Wilson line and the brane position. A stack of D7-branes sitting on the divisor preserves the supersymmetry and, in this paper, we will use 4-cycle as the same meaning of divisor.
} 
volume is given by $\tau_{2}$, and hence one will find that $a=2 \pi / N$ and $b=2 \pi / M$, in which $N$ and $M$ are the rank of the gauge group, are of order unity. Hence the relevant holomorphic gauge coupling $f$ on the branes are given by $\tau_{2}$, and is supposed to be corrected by the Euclidean D3-instanton effects wrapping on non-rigid 4-cycle, whose volume is $\tau_{3}: f_{i}=$ $T_{2}+C_{i} e^{-2 \pi T_{3}}+$ dilaton. Non-perturbative effect with such a gauge coupling $e^{-f_{i}}$ is called poly-instanton [49]. ${ }^{9}$ Note that we set $\left\langle W_{\text {flux }}\right\rangle=\left\langle\int G_{3} \wedge \Omega\right\rangle=0$ and it seems that the number of such flux vacua is not too small [55]. ${ }^{10}$ Here $G_{3}$ is the three-form flux and $\Omega$ is holomorphic three-form on the relevant Calabi-Yau space.

The supergravity potential is written by

$$
V_{F}=e^{K}\left[K^{I \bar{J}} D_{I} W\left(D_{J} W\right)^{\dagger}-3|W|^{2}\right], \quad \text { where } D_{I} W=\partial_{I} W+W\left(\partial_{I} K\right) .
$$

Here $K^{I \bar{J}}$ is the inverse of the Kähler metric $K_{I \bar{J}}=\partial_{I} \partial_{\bar{J}} K$.

One can easily check the vacuum structure. ${ }^{11}$ First we define the gravitino mass

$$
m_{3 / 2}=e^{K / 2} W \sim \frac{\left\langle W_{\text {eff }}\right\rangle}{\mathcal{V}}
$$

and $F$-terms of SUSY-breaking order parameters are given by

$$
F^{I}=-e^{K / 2} K^{I \bar{J}}\left(D_{J} W\right)^{\dagger} .
$$

The heaviest modulus $T_{2}$ is stabilized near supersymmetric location like the racetrack model: ${ }^{12}$

$$
\begin{aligned}
& F^{T_{2}} \sim \partial_{T_{2}} W \sim \partial_{T_{2}} W_{\mathrm{eff}} \sim 0 \quad \rightarrow \quad\left\langle T_{2}\right\rangle \simeq \frac{1}{a-b} \log \left(\frac{a A}{b B}\right), \\
& m_{\tau_{2}} \simeq m_{\sigma_{2}} \sim\left\langle W_{\text {eff }}\right\rangle \ll 1, \quad \frac{F^{T_{2}}}{2 \tau_{2}} \sim m_{3 / 2} \frac{m_{3 / 2}}{m_{\tau_{2}}} .
\end{aligned}
$$

Next, the $T_{3}$ will be also fixed near the supersymmetric solution like the minimal LVS [17]:

$$
\begin{gathered}
F^{T_{3}} \sim 0 \rightarrow \mathcal{V} \sim \tau_{1}^{3 / 2} \sim \frac{W_{\text {eff }}}{A_{\text {eff }}} e^{2 \pi T_{3}} \\
m_{\tau_{3}} \simeq m_{\sigma_{3}} \sim \log (\mathcal{V}) m_{3 / 2} \sim \log (\mathcal{V}) \frac{\left\langle W_{\text {eff }}\right\rangle}{\mathcal{V}}, \quad \frac{F^{T_{3}}}{2 \tau_{3}} \sim m_{3 / 2} \frac{m_{3 / 2}}{m_{\tau_{3}}} .
\end{gathered}
$$

\footnotetext{
${ }^{9}$ In general, there would be a case that an instanton wrapping on the non-rigid 4-cycle would contribute not to the superpotential directly but to the gauge coupling on a brane wrapping on the rigid cycle, because of too many fermionic zero modes on the non-rigid brane. In such a case, the modulus relevant to the nonrigid cycle would be stabilized via poly-instanton, i.e. gaugino condensations/instantons, generated on the rigid brane. (However, K3 divisor is available only if there are some additional mechanisms on it, e.g. three form fluxes [50].) Furthermore, this effect becomes important for generating larger mass scale hierarchy between moduli rather than those of usual instantons [51-54].

${ }^{10}$ In the literature, it was found that $N_{\text {vacua }}\left(W_{\text {flux }}=0\right) / N_{\text {vacua }} \sim 1 / L^{\mathcal{D} / 2}$, where $L$ is the upper limit of flux quanta and $\mathcal{D}$ is an integer. Cases with $L=\mathcal{O}\left(10-10^{3}\right)$ (depending on the RR-tadpole cancellation) and $\mathcal{D}=\mathcal{O}(1)$ were considered there.

${ }^{11}$ We numerically checked the followings are correct.

${ }^{12}$ See, for instance, [56] for an explanation about the F-term structures.
} 
Finally, the lightest modulus $T_{1}$, which is no-scale modulus corrected by the nonperturbative superpotential and $\xi \mathcal{V}^{-1}$ in the Kähler potential, will be stabilized because of the $\xi$-dependent term like the minimal LVS case:

$$
\left\langle T_{3}\right\rangle \sim \frac{\left\langle\log \left(\tau_{1}^{3 / 2}\right)\right\rangle}{2 \pi} \sim \xi^{2 / 3}, \quad m_{\tau_{1}} \sim \frac{\left\langle W_{\text {eff }}\right\rangle}{\sqrt{\log (\mathcal{V})} \mathcal{V}^{3 / 2}}, \quad \frac{F^{\tau_{1}}}{2 \tau_{1}}=m_{3 / 2}\left(1+\mathcal{O}\left(\log (\mathcal{V})^{-1} \mathcal{V}^{-1}\right)\right)
$$

Note that $\sigma_{1}$ is massless axion and modulino $\tilde{T}_{1}$ is the goldstino. Thus one obtains $\mathcal{V} \simeq$ $\tau_{1}^{3 / 2} \gg \tau_{2}^{3 / 2} \sim \tau_{3}^{3 / 2}$. Because of the large volume, even if there is a superpotential $\delta W \sim$ $e^{-2 \pi T_{1}}, \sigma_{1}$ still stays almost massless. As a result, the vacuum is non-supersymmetric AdS:

$$
\left\langle V_{F}\right\rangle \sim-\left\langle\frac{\left|W_{\text {eff }}\right|^{2}}{\log (\mathcal{V}) \mathcal{V}^{3}}\right\rangle<0
$$

Following the KKLT proposal $[43,57]$, the uplifting term is required to obtain the tiny cosmological constant $\langle V\rangle \approx 0$, where

$$
\begin{aligned}
V & =V_{F}+V_{\text {uplift }}, \\
V_{\text {uplift }} & =\epsilon e^{2 K / 3}=\frac{\epsilon}{\mathcal{V}^{4 / 3}}, \quad \epsilon \sim\left\langle\frac{|W|^{2}}{\log (\mathcal{V}) \mathcal{V}^{5 / 3}}\right\rangle \ll 1 .
\end{aligned}
$$

Here we have assumed that $V_{\text {uplift }}$ is generated on the sequestered anti-D3-branes on a top of the warped throat ${ }^{13}$ and $\epsilon$ means the minimum of the warp factor.

Let us consider to include the dilaton and complex structure moduli. They will be stabilized, being consistent with the Kähler moduli $T_{i}$, because the potential for them is of $\mathcal{O}\left(\mathcal{V}^{-2}\right)$ with $W_{\text {flux }}$ whereas the Kähler moduli are done at $\mathcal{O}\left(\mathcal{V}^{-1}\right)$ for $T_{2}$ and at $\mathcal{O}\left(\mathcal{V}^{-3}\right)$ for $T_{1}$ and $T_{3}$. Then, their mass scale will be of order $M_{\mathrm{pl}} / \mathcal{V}$. In this case, the contribution from the dilaton $S$ and complex structure moduli $U$ is written as

$$
K_{\text {moduli }}=-\log \left(S+S^{\dagger}\right)+K_{T}\left(T+T^{\dagger}\right)+K_{U}\left(U+U^{\dagger}\right) .
$$

And then

$$
m_{S, U} \sim \frac{W_{\text {flux }}^{\prime \prime}}{K^{\prime \prime}} e^{K / 2} \sim \frac{1}{\mathcal{V}}
$$

Here note that the supersymmetric mass terms between $S$ and $U$ are generally included in $W_{\text {flux }}$. In this section, we assume that $F^{U}=0$ and

$$
\left\langle F^{S}\right\rangle \sim \frac{1}{\mathcal{V}}\left(\left(\partial_{S} K_{\text {moduli }}\right)\langle W\rangle+\partial_{S} W_{\text {flux }}\right) \sim \frac{\left(\partial_{S} K_{T}\right)}{\log (\mathcal{V}) \mathcal{V}}\left\langle W_{\text {eff }}\right\rangle \sim \frac{\left\langle W_{\text {eff }}\right\rangle}{\log (\mathcal{V}) \mathcal{V}^{2}}
$$

This is because one expects that $S$ would be stabilized almost without $\xi$-dependent Kähler potential: $-W /\left(S+S^{\dagger}\right)+\partial_{S} W_{\text {flux }} \approx 0$, though its magnitude would depend on flux models strongly [65, 66]. Hence we will not consider also the derivative of $F^{S}$ with respect to any moduli.

\footnotetext{
${ }^{13}$ Dynamical SUSY breaking model is also viable [48]. See also [58-63] for the KKLT case and discussion on such an anti-brane from the view point of the 10D supergravity [64].
} 


\subsubsection{Visible sector}

Next, let us focus on the visible sector in this local model, and suppose that the minimal supersymmetric standard model (MSSM) is localized on the rigid D7-brane wrapping on the $\tau_{2} 4$-cycle. Therefore the relevant effective potential will be given by $[67,68]^{14}$

$$
K_{\mathrm{vis}}=\frac{\left(T_{2}+T_{2}^{\dagger}\right)^{\lambda_{i}}}{\left(T_{1}+T_{1}^{\dagger}\right)}\left|\phi_{i}\right|^{2}=Z_{i}\left|\phi_{i}\right|^{2}, \quad f_{\mathrm{vis}, a}=\frac{1}{4 \pi}\left(T_{2}+h_{a} S\right), \quad W_{\mathrm{vis}}=W_{\mathrm{MSSM}}\left(\phi_{\mathrm{vis}}\right) .
$$

Here we will take a minimal model $\lambda_{i}=1 / 3$ and assume the approximate gauge coupling unification, i.e. $h_{a}=\mathcal{O}(1)(a=1,2,3)$, which is given by the world volume flux depending on the gauge group on the visible brane [69]. For instance $h_{2}<h_{3}<h_{1}$ is possible, depending on the model. Notice that the gauge fluxes on the visible brane are relevant for obtaining the chiral matter spectra. $S=1 / g_{s}-i C_{0}^{\mathrm{RR}}$, where $C_{0}^{\mathrm{RR}}$ is the RR scalar zero mode, is the $4 \mathrm{D}$ string axion-dilaton fixed by closed string flux. The factor $1 /\left(T_{1}+T_{1}^{\dagger}\right)^{-1}$ is important because the physical couplings in the superpotential are (almost) independent on the $T_{1}$ in the large $\mathcal{V}$ limit:

$$
\begin{aligned}
W=y \frac{\phi_{\mathrm{vis}}^{n}}{M_{\mathrm{pl}}^{n-3}} \rightarrow W_{\text {global }} & =e^{K_{\text {moduli }} / 2} \frac{y}{\sqrt{Z^{3}}} \frac{\phi_{\mathrm{vis}}}{\sqrt{Z^{n-3}} M_{\mathrm{pl}}^{n-3}} \\
& =y^{\text {phys }} \frac{\phi_{c}^{n}}{M_{*}^{n-3}} .
\end{aligned}
$$

Thus one finds $y^{\text {phys }}=y\left(\tau_{2}\right)$ and

$$
M_{*} \simeq \frac{M_{\mathrm{pl}}}{\mathcal{V}^{1 / 3}} \sim M_{\mathrm{wind}}
$$

The cutoff scale in the superpotential would be of order winding mode scale [38-41, 70]. Therefore we will not consider the moduli-redefinition effect [71] in this whole paper.

The SUSY-breaking soft masses at the messenger scale are given by ${ }^{15}$ (see, e.g. [72])

$$
\begin{aligned}
m_{0}^{2} & \simeq-F^{I}\left(F^{\bar{J}}\right)^{\dagger} \partial_{I} \partial_{\bar{J}} \log \left(e^{-K_{\text {moduli }} / 3} Z\right) \\
& \sim \frac{1}{\mathcal{V}}\left|\frac{F^{T_{1}}}{2 \tau_{1}}\right|^{2} \simeq \frac{m_{3 / 2}^{2}}{\mathcal{V}} \sim \frac{\left\langle\left|W_{\text {eff }}\right|^{2}\right\rangle}{\mathcal{V}^{3}} \\
M_{a} & \simeq F^{I} \partial_{I} \log \left(f_{\text {vis }, a}\right)+\frac{\alpha_{a}}{4 \pi}\left(b_{a} F^{\varphi}-2 \sum_{i} \operatorname{tr}\left(T_{a}^{2}\left(\phi_{i}\right)\right) F^{I} \partial_{I} \log \left(e^{-K_{\text {moduli }} / 3} Z_{i}\right)\right) \\
& \simeq h_{a} \frac{F^{S}}{2 \tau_{2}}+\frac{\alpha_{a}}{4 \pi} \frac{1}{\mathcal{V}} \frac{F^{T_{1}}}{2 \tau_{1}} \sim \frac{m_{3 / 2}}{\log (\mathcal{V}) \mathcal{V}} \sim \frac{\left\langle W_{\text {eff }}\right\rangle}{\log (\mathcal{V}) \mathcal{V}^{2}},
\end{aligned}
$$

\footnotetext{
${ }^{14}$ In this case, an instanton on $\tau_{3}$ would contribute to the visible gauge coupling while the gaugino condensations on $\tau_{2}$ would have effect on the visible small Yukawa coupling. However, we neglected those effects for a simplicity. The contribution of an instanton effect $4 \pi \delta f_{\text {vis }}=e^{-2 \pi T_{3}}$ in the holomorphic gauge coupling to the gaugino mass will be the same order as computation shown below at most. For non-perturbative Yukawa coupling, even if there would be terms $\delta W_{\text {Yukawa }}=e^{-a T_{2}} \phi^{3}$ or $e^{-a T_{2}} e^{-2 \pi T_{3}} \phi^{3}$ from gaugino condensations, one will not suffer from the flavor changing neutral current induced by nonperturbative $A$-term because $m_{0}$ is large and $F^{T_{2}}$ is small.

${ }^{15} \mathrm{We}$ added $2\left\langle V_{\text {uplift }}\right\rangle / 3$ contribution to the soft scalar mass from the uplifting potential.
} 


$$
\begin{aligned}
A_{i_{1} \cdots i_{n}} & \simeq(n-3) F^{\varphi}-F^{I} \partial_{I} \log \left(\frac{y_{i_{1} \cdots i_{n}}}{e^{-n K_{\text {moduli }} / 3} Z_{i_{1}} \cdots Z_{i_{n}}}\right) \\
& \sim \frac{1}{\mathcal{V}} \frac{F^{T_{1}}}{2 \tau_{1}}+\frac{F^{S}}{S+S^{\dagger}} \sim \frac{m_{3 / 2}}{\mathcal{V}} \sim \frac{\left\langle W_{\text {eff }}\right\rangle}{\mathcal{V}^{2}} .
\end{aligned}
$$

Here the second term in the gaugino mass is originating from the anomaly mediation; $b_{a}(a=1,2,3)$ is 1-loop coefficient of beta function of the gauge coupling in the MSSM and $\operatorname{tr}\left(T_{a}^{2}\left(\phi_{i}\right)\right)$ is dynkin index for the matter $\phi_{i}$ charged under the MSSM gauge group. Note that the anomaly mediation contribution would be suppressed or be at most the same order compared to $F^{S}$ in the gaugino mass, because the F-term of the compensator

$$
F^{\varphi}=m_{3 / 2}+\frac{\partial_{I} K}{3} F^{I} \sim \frac{F^{S}}{S+S^{\dagger}}+\frac{m_{3 / 2}}{\log (\mathcal{V}) \mathcal{V}} \sim \frac{\left\langle W_{\text {eff }}\right\rangle}{\log (\mathcal{V}) \mathcal{V}^{2}}
$$

will be also suppressed.

The above results of the soft masses are easily understood: Because $F^{T_{2}}$ is negligible due to the very massive $T_{2}$, SUSY-breaking structure in the visible sector is determined by the no-scale modulus $T_{1}$ with $\mathcal{V}^{-1}$ corrections in the potential. (However, in the computation of the coupling between the gauginos and moduli, the $F^{T_{2}}$ is important.) Note that the perturbative Peccei-Quinn symmetries of moduli/dilaton $T, S \rightarrow T, S+$ const. forbid the moduli/dilaton dependence of couplings $y_{i_{1} \cdots i_{n}}$ in the visible superpotetial.

Finally the mass spectrum is summarized as

$$
m_{\tau_{2}}>m_{S, U} \gg m_{\tau_{3}}>m_{3 / 2}>m_{0} \gtrsim m_{\tau_{1}} \gg A_{n}>M_{a} .
$$

This is a realization of split SUSY [73, 74]. What is important is that the decay of the lightest modulus to gravitinos is kinematically forbidden because of the fact that $m_{3 / 2} \gg$ $m_{\tau_{1}}$. Recall that axion $\sigma_{1}$ is massless.

\subsubsection{A numerical example based on the model 2 in the literature [45]}

Following the literature, we hereafter will use the following parameters for numerical calculations

$$
\begin{aligned}
& \eta_{1}=1, \quad \eta_{2}=\frac{1}{53}, \\
& \eta_{3}=\frac{1}{6} \\
& \chi=-136, \quad g_{s}=\frac{2}{5}, \\
& C_{1}=1, \\
& C_{2}=3 \text {, } \\
& a=\frac{2 \pi}{8}, \quad b=\frac{2 \pi}{9}, \\
& A=1.28 \times 10^{5} \text {, } \\
& B=1.6 \times 10^{4} \text {. }
\end{aligned}
$$

$A$ and $B$ are much larger than unity, however this fact may be explained in terms of gaugino condensation on the magnetized D7-brane [75, 76]:

$$
A \sim e^{-a\left(h_{A} / g_{s}\right)} \gg 1, \quad B \sim e^{-b\left(h_{B} / g_{s}\right)} \gg 1 .
$$

Here $h_{A, B} \sim \frac{-1}{4 \pi^{2}} \int_{\Sigma_{A, B}} F_{A, B} \wedge F_{A, B}-\mathcal{R}_{A, B} \wedge \mathcal{R}_{A, B}$ is assumed to be negative and be of $\mathcal{O}(1)$. $\Sigma_{A, B}$ are respectively the 4-cycle worldvolume in the extra dimension of two branes $(A, B)$ which experience gaugino condensations. $F_{A, B}$ and $\mathcal{R}_{A, B}$ are magnetic flux and geometric 


\begin{tabular}{|c|c|c|c|}
\hline Fundamental parameters & Moduli masses & F-terms & Soft masses \\
\hline$M_{\text {string }}=1.2 \times 10^{16} \mathrm{GeV}$ & $m_{S, U}=3.4 \times 10^{13} \mathrm{GeV}$ & $F^{S}=\mathcal{O}\left(10^{3}\right) \mathrm{GeV}$ & $m_{3 / 2}=1.4 \times 10^{9} \mathrm{GeV}$ \\
$M_{*}=7.9 \times 10^{16} \mathrm{GeV}$ & $m_{\tau_{2}, \sigma_{2}}=3.1 \times 10^{17} \mathrm{GeV}$ & $F^{T_{2}} / 2 \tau_{2}=\mathcal{O}(10) \mathrm{GeV}$ & $m_{0}=\mathcal{O}\left(10^{6}-10^{7}\right) \mathrm{GeV}$ \\
$\left|W_{\mathrm{eff}}\right| / M_{\mathrm{pl}}^{3}=4.1 \times 10^{-5}$ & $m_{\tau_{3}, \sigma_{3}}=5.3 \times 10^{10} \mathrm{GeV}$ & $F^{T_{3}} / 2 \tau_{3}=2.9 \times 10^{7} \mathrm{GeV}$ & $A_{n}=\mathcal{O}\left(10^{3}-10^{4}\right) \mathrm{GeV}$ \\
$\langle\mathcal{V}\rangle=78559$ & $m_{\tau_{1}}=1.5 \times 10^{6} \mathrm{GeV}$ & $F^{T_{1}} / 2 \tau_{1}=1.4 \times 10^{9} \mathrm{GeV}$ & $M_{1 / 2}=\mathcal{O}\left(10^{3}\right) \mathrm{GeV}$ \\
\hline
\end{tabular}

Table 1. A numerical example. Here we have included the dilaton dependence $e^{K} \supset 1 / 2 g_{s}$ in the supergravity potential. Although $m_{\tau_{2}}$ is greater than the string scale, it is smaller than the Planck scale; this model could be valid just as the supergravity model. We assumed $F^{S} \sim F^{\varphi}=O(1-10)$ $\mathrm{TeV}$. The magnitude of $F^{T_{1}}$ strongly depends on the accuracy of computation because $T_{1}$ is so heavy. Note that $\sigma_{1}$ is massless axion decoupled from the visible sector.

curvature two-form on the relevant two branes. In this example, in spite of $A, B \gg 1$, one finds

$$
\left\langle W_{\text {eff }}\right\rangle \ll 1
$$

and hence the scales of gaugino condensations is lower than the cutoff scale. Notice that $C_{i}=\mathcal{O}(1)(i=1,2)$ will be also plausible when the instanton on $\tau_{3}$ is not fluxed and the geometric curvatures relevant to $\tau_{3}$ are zero.

With these numerical parameters, the vacuum expectation values of moduli are read as

$$
\langle\mathcal{V}\rangle=78559, \quad\left\langle T_{2}\right\rangle=25.18, \quad\left\langle T_{3}\right\rangle=2.88
$$

Other parameters at the vacuum is shown in table 1 . Although $m_{\tau_{2}}$ exceeds the string scale in this numerical example as shown, we are going to continue the discussion; it is expected that a proper choice of parameters would bring us to a viable region in which $m_{\tau_{2}}$ is below the string scale without changing the essence. In addition, we do not include complex structure moduli dependence in the scalar potential of $e^{K}$ in the numerical computation. This factor would reduce the masses slightly. At any rate, it is smaller than the Planck scale and hence this model could be valid just as the supergravity model.

\subsubsection{Higgs sector: $\mu / B \mu$-term}

So far we have studied the moduli sector and the visible sector except for Higgs fields $\left(H_{u}, H_{d}\right)$ in the MSSM. In the Higgs sector, generation of $\mu / B \mu$-term is important. Furthermore, because SUSY-breaking stop mass is so heavy as $10^{6} \mathrm{GeV}$ in this model, the Higgs quartic coupling $\lambda_{\text {tree }} \simeq\left(g_{2}^{2}+g_{Y}^{2}\right) \cos ^{2}(2 \beta) / 8$ at a high scale should be almost vanishing to obtain $m_{\text {Higgs }} \simeq 125 \mathrm{GeV}$ for the lightest Higgs boson. In other words, $\tan \beta=\mathcal{O}(1)$ is required [77]. This can be naturally explained if the approximate shift symmetry exists in the Higgs fields [78].

If $\left(H_{u}, H_{d}\right)$ have the origin from a higher dimensional gauge field (SUSY gauge-Higgs unification) through the gauge symmetry breaking, e.g. by boundary condition, one would find an approximate shift symmetry $H_{u, d} \rightarrow H_{u, d}+i \gamma$, which comes from the gauge symmetry. Here $\gamma$ is the constant. This constrains the structure of their leading Kähler potential at the tree level, though this symmetry will be broken at the quantum level or by the 
coupling to matter fields at the tree level (including worldsheet instantons). As a result the Kähler potential will be then given by

$$
K_{\text {Higgs }}=\frac{\left(T_{2}+T_{2}^{\dagger}\right)^{1 / 3}}{\left(T_{1}+T_{1}^{\dagger}\right)}\left|H_{u}+H_{d}^{\dagger}\right|^{2}+\cdots .
$$

We have omitted terms which are breaking such shift symmetry, and it can be expected that they are threshold correction from the massive modes, e.g. KK modes. What is important is that the Giudice-Masiero term is included:

$$
K_{\text {Higgs }} \supset \frac{\left(T_{2}+T_{2}^{\dagger}\right)^{1 / 3}}{\left(T_{1}+T_{1}^{\dagger}\right)} H_{u} H_{d} .
$$

From this Kähler potential, the proper $\mu$-term and $B \mu$-term are obtained

$$
\begin{aligned}
\mu & \simeq m_{3 / 2}-\frac{F^{T_{1}}}{2 \tau_{1}} \sim \frac{m_{3 / 2}}{\log (\mathcal{V}) \mathcal{V}} \sim \frac{\left\langle W_{\text {eff }}\right\rangle}{\log (\mathcal{V}) \mathcal{V}^{2}}=\mathcal{O}(1) \mathrm{TeV} \\
B \mu & \simeq|\mu|^{2}+m_{0}^{2}=\mathcal{O}\left(10^{12}\right) \mathrm{GeV}^{2}
\end{aligned}
$$

Here we have added $2\left\langle V_{\text {uplift }}\right\rangle / 3$ contribution to $B \mu$-term and used values in the numerical example. Although we ignored $F^{T_{2}}$ in the soft masses, this becomes important for computation of the moduli coupling. From the extremum condition of the Higgs potential at the electroweak scale, one will find

$$
\sin (2 \beta)=\frac{2 B \mu}{m_{H u}^{2}+m_{H d}^{2}+2|\mu|^{2}}=\mathcal{O}(1) .
$$

It is expected the correction term will come from quantum effects and the threshold correction mentioned above. Thus $\tan \beta=\mathcal{O}(1)$ will be realized. Note that a fine-tuning is now demanded for achieving the correct electroweak symmetry breaking.

\subsection{Moduli problem in LVS}

Here let us discuss the moduli problem, focusing on the lightest modulus. The purpose of this subsection is to calculate the dilution factor by the lightest modulus decay.

Before uplifting the scalar potential, the AdS minimum has the depth of order $-\left|\left\langle W_{\text {eff }}\right\rangle\right|^{2} \log (\mathcal{V})^{-1} \mathcal{V}^{-3} \simeq-m_{\tau_{1}}^{2} M_{\mathrm{pl}}^{2}$. The height of the potential barrier is comparable to the depth. Hence, in order to avoid run-away and decompactification, the Hubble parameter during the inflation is constrained as [30]

$$
H_{\text {inf }} \lesssim m_{\tau_{1}}
$$

This is because the inflaton potential energy $3 H_{\mathrm{inf}}^{2} M_{\mathrm{pl}}^{2}$ should be smaller than the height of the barrier $m_{\tau_{1}}^{2} M_{\mathrm{pl}}^{2}$ (see also [79]). ${ }^{16}$ For an inflation model with $H_{\mathrm{inf}} \lesssim m_{\tau_{1}}$, the heavier

\footnotetext{
${ }^{16}$ The constraint may be modified, if the position or the potential of moduli is changed drastically during the inflation. For instance, one can consider cases that a field shifted by the inflaton VEV makes the higher barrier or inflaton is the lightest rolling modulus [30], realizing $m_{\tau_{1}}<H_{\text {inf }}$. (See also the modulus inflation model realizing $m_{3 / 2}<H_{\text {inf }}$ for non-LVS case [80, 81].) Hence, we expect that coherent oscillation of moduli with a large amplitude could occur in LVS, depending on the inflation model for realizing $c^{2}>0$.
} 
moduli will stay at the true minima during inflation, while the lightest one may be deviated from the true minimum. The shift of the modulus VEV during inflation is expected to be of order

$$
\frac{H_{\mathrm{inf}}^{2}}{m_{\tau_{1}}^{2}} M_{\mathrm{pl}}
$$

in terms of the canonically normalized modulus. Note however that it does not necessarily mean that the lightest modulus starts to oscillate with an amplitude given above. If the upper bound on $H_{\text {inf }}$ is saturated, i.e., $H_{\mathrm{inf}} \sim m_{\tau_{1}}$, there is a cosmological moduli problem as usual; the modulus $\tau_{1}$ starts to oscillate with an amplitude of order the Planck scale, and dominates the Universe soon after the reheating. The situation is a bit more complicated when $H_{\text {inf }}<m_{\tau_{1}}$. At the end of inflation, the inflaton begins to oscillate and the potential energy of the inflaton is transferred to the kinetic energy. If the time scale of the inflation oscillation is shorter than that of the modulus, i.e. $m_{I} \gg m_{\tau_{1}}$, where $m_{I}$ is the inflaton mass around true vacuum, the modulus cannot follow the change of the potential caused by the inflaton and it starts to oscillate around the true vacuum soon after the inflation ends [82].

Thus, the oscillation amplitude of the modulus is expected to be of order $\frac{H_{\text {inf }}^{2}}{m_{\tau_{1}}^{2}} M_{\mathrm{pl}}<$ $M_{\mathrm{pl}}$, when the whole potential energy of inflaton is transmitted into the kinetic energy. Noting that the inflation scale is also bounded below for the AD mechanism to work, $H_{\text {inf }}>$ $m_{0} / c$, the resultant moduli abundance can be sizable. In particular, for the numerical example shown in table 1 , the inflation scale is almost comparable to $m_{\tau_{1}}$, and so, there is a serious cosmological moduli problem.

On the other hand, if all the time scale of the inflaton dynamics is longer than that of the modulus, i.e. $m_{I}, H_{\text {inf }}<m_{\tau_{1}}$, the modulus would settle down to the true vacuum without oscillation and there will be no moduli problem. However, the reheating temperature by inflaton decay tends to be low for such low-scale inflation models, and so, the AD mechanism will be an important possibility at any rate.

To summarize, as long as the inflaton mass at the potential minimum is heavier than the modulus mass, the modulus starts coherent oscillations soon after inflation. Although the oscillation amplitude is suppressed for a lower inflation scale, it is bounded below for the successful AD baryogenesis. Therefore, the low-energy theory suffers from the cosmological moduli problem. In the following we consider the cosmology of the modulus, assuming that it dominates the energy density of the Universe.

Here let us express the moduli fields in terms of the canonically normalized mass eigenstates $\{\delta \phi, \delta a\}: 17$

$$
\left(\begin{array}{c}
\delta \tau_{1} \\
\delta \tau_{2} \\
\delta \tau_{3} \\
\delta\left(1 / g_{s}\right)
\end{array}\right) \sim\left(\begin{array}{c}
\mathcal{V}^{2 / 3} \\
\frac{m_{3 / 2}}{m_{\tau_{2}}} \\
\frac{m_{3 / 2}}{m_{\tau_{3}}} \\
\mathcal{V}^{-1 / 2}
\end{array}\right) \delta \phi_{1}+\left(\begin{array}{c}
\mathcal{V}^{1 / 6} \\
\mathcal{V}^{1 / 2} \\
\mathcal{V}^{-1 / 2} \\
\mathcal{V}^{-1}
\end{array}\right) \delta \phi_{2}+\left(\begin{array}{c}
\mathcal{V}^{1 / 6} \\
\mathcal{V}^{-1 / 2} \\
\mathcal{V}^{1 / 2} \\
\mathcal{V}^{-1}
\end{array}\right) \delta \phi_{3}+\left(\begin{array}{c}
\mathcal{V}^{1 / 6} \\
\mathcal{V}^{-1 / 2} \\
\mathcal{V}^{-1 / 2} \\
\mathcal{O}(1)
\end{array}\right) \delta \phi_{s}
$$

\footnotetext{
${ }^{17}$ We have checked this relation numerically. This is consistent with the results studied in [83] and [84].
} 


$$
\left(\begin{array}{c}
\delta \sigma_{1} \\
\delta \sigma_{2} \\
\delta \sigma_{3} \\
\delta C_{0}^{\mathrm{RR}}
\end{array}\right) \sim\left(\begin{array}{c}
\mathcal{V}^{2 / 3} \\
0 \\
0 \\
0
\end{array}\right) \delta a_{1}+\left(\begin{array}{c}
\mathcal{V}^{1 / 6} \\
\mathcal{V}^{1 / 2} \\
\mathcal{V}^{-1 / 2} \\
\mathcal{V}^{-1}
\end{array}\right) \delta a_{2}+\left(\begin{array}{c}
\mathcal{V}^{1 / 6} \\
\mathcal{V}^{-1 / 2} \\
\mathcal{V}^{1 / 2} \\
\mathcal{V}^{-1}
\end{array}\right) \delta a_{3}+\left(\begin{array}{c}
0 \\
\mathcal{V}^{-1 / 2} \\
\mathcal{V}^{-1 / 2} \\
\mathcal{O}(1)
\end{array}\right) \delta a_{s}
$$

The mass eigenstates $\delta \phi_{i}$ should not be confused with the $\mathrm{AD}$ field $\phi$. Note that $m_{3 / 2} / m_{\tau_{2}} \sim 1 / \mathcal{V}$ and $m_{3 / 2} / m_{\tau_{3}} \sim 1 / \log (\mathcal{V})$. Here $\left(\delta \phi_{2}, \delta a_{2}\right),\left(\delta \phi_{3}, \delta a_{3}\right),\left(\delta \phi_{s}, \delta a_{s}\right)$ and $\delta \phi_{1}$ have mass eigenvalues of $m_{\tau_{2}}, m_{\tau_{3}}, m_{S}$ and $m_{\tau_{1}}$, respectively, while $\delta a_{1}$ is massless.

Let us estimate the lifetime of $\phi_{1}$. The relevant moduli couplings to the visible sector arise from the gaugino mass and $\mu$-term:

$$
\lambda_{c} \lambda_{c}\left[\frac{F^{T_{2}}}{2 \tau_{2}}+\text { anomaly-mediated terms }\right]+\frac{1}{3} \frac{F^{T_{2}}}{2 \tau_{2}} \tilde{h}_{c} \tilde{h}_{c},
$$

where $\lambda_{c}$ and $\tilde{h}_{c}$ are canonically normalized gaugino and higgsino. The derivative of $F^{T_{2}}$ with respect to the moduli are given by

$$
\left(\begin{array}{l}
\partial_{T_{1}} \\
\partial_{T_{2}} \\
\partial_{T_{3}}
\end{array}\right) \frac{F^{T_{2}}}{2 \tau_{2}} \sim\left(\begin{array}{l}
\frac{m_{3 / 2}}{\mathcal{V}^{2 / 3}} \\
m_{\tau_{2}} \\
m_{\tau_{3}}
\end{array}\right) .
$$

From this, one can read the interactions relevant to the decay of the $\delta \phi_{1}$ :

$$
\delta \phi_{1} \frac{m_{3 / 2}}{M_{\mathrm{pl}}}\left[\lambda_{c} \lambda_{c}+\tilde{h}_{c} \tilde{h}_{c}\right] .
$$

Due to the loop factor, the anomaly mediation contribution to the gaugino mass is irrelevant for the coupling between the modulus and gauginos. Thus the modulus decays into gauginos and higgsinos if it is allowed kinematically, and the decay width to the visible sector is given by ${ }^{18}$

$$
\begin{aligned}
\Gamma_{\phi_{1}} & \simeq \Gamma\left(\phi_{1} \rightarrow \tilde{g} \tilde{g}\right)+\Gamma\left(\phi_{1} \rightarrow \tilde{h} \tilde{h}\right) \\
& \sim \frac{N_{c}}{4 \pi}\left(\frac{m_{3 / 2}}{M_{\mathrm{pl}}}\right)^{2} m_{\tau_{1}} \sim \frac{\mathcal{V} \log (\mathcal{V})}{4 \pi} \frac{m_{\tau_{1}}^{3}}{M_{\mathrm{pl}}^{2}} \sim \frac{\left\langle W_{\mathrm{eff}}\right\rangle^{3}}{\sqrt{\log (\mathcal{V})} \mathcal{V}^{7 / 2}} M_{\mathrm{pl}} .
\end{aligned}
$$

Here $N_{c}(=12)$ is the effective number of decay channel. Note that the decay of the lightest modulus to gravitinos is kinematically forbidden. Here, the decay of $\phi_{1} \rightarrow 2 a_{1}$ also proceeds via the kinetic term

$$
\frac{\delta \phi_{1}}{M_{\mathrm{pl}}}\left(\partial \delta a_{1}\right)^{2}
$$

The branching fraction of the decay to a pair of axions is estimated as

$$
B\left(\phi_{1} \rightarrow a_{1} a_{1}\right) \simeq\left(\frac{m_{\tau_{1}}}{m_{3 / 2}}\right)^{2} \sim \frac{1}{\log (\mathcal{V}) \mathcal{V}} \sim 10^{-6} .
$$

Thus the produced axions from the modulus decay is too small to affect the BBN or CMB. ${ }^{19}$

\footnotetext{
${ }^{18}$ We will use the dimensionless $W_{\text {eff }}$ through the normalization of $W_{\text {eff }} \rightarrow M_{\mathrm{pl}}^{3} W_{\text {eff }}$.

${ }^{19}$ The present observations give a slight preference to the existence of dark radiation. The non-thermal production of axions is an interesting possibility [85, 86].
} 
The decay temperature of $\delta \phi_{1}$ in the radiation dominated Universe is estimated as

$$
T_{\mathrm{dec}}^{\phi_{1}} \simeq \sqrt{\Gamma_{\phi_{1}} M_{\mathrm{pl}}} \simeq 1.1 \times 10^{3} \mathrm{GeV}\left(\frac{m_{\tau_{1}}}{1.5 \times 10^{6} \mathrm{GeV}}\right)^{1 / 2}\left(\frac{m_{3 / 2}}{1.4 \times 10^{9} \mathrm{GeV}}\right) .
$$

Thus, the lightest modulus $\phi_{1}$ decays well before the BBN.

The dilution factor due to the modulus decay can be estimated as follows. For most of the parameter region of our interest, the $\delta \phi_{1}$ starts to oscillate soon after inflation. Hence the temperature when the energy density of $\delta \phi_{1}$ dominates the Universe is given by

$$
T_{\mathrm{dom}}^{\phi_{1}} \simeq \frac{T_{R}}{3}\left(\frac{\Delta \phi_{1}}{M_{\mathrm{pl}}}\right)^{2} .
$$

$\Delta \phi_{1}$ is the oscillation amplitude of the lightest modulus around the true minimum: $\Delta \phi_{1} / M_{\mathrm{pl}} \sim H_{\mathrm{inf}}^{2} / m_{\tau_{1}}^{2}$ as mentioned above. Thus the dilution factor by the $\delta \phi_{1}$ decay is given by

$$
\Delta^{-1} \simeq \frac{T_{\mathrm{dec}}^{\phi_{1}}}{T_{\mathrm{dom}}^{\phi_{1}}} \simeq 3.4 \times 10^{-4}\left(\frac{m_{\tau_{1}}}{1.5 \times 10^{6} \mathrm{GeV}}\right)^{1 / 2}\left(\frac{m_{3 / 2}}{1.4 \times 10^{9} \mathrm{GeV}}\right)\left(\frac{T_{R}}{10^{7} \mathrm{GeV}}\right)^{-1}\left(\frac{\Delta \phi_{1}}{M_{\mathrm{pl}}}\right)^{-2} .
$$

Finally we briefly comment on the neutralino dark matter non-thermally produced by the decay of the lightest modulus. We assume that the LSP is Wino or higgsino-like neutralino (or their combination). Although a large number of the neutralino LSP is produced by the modulus decay, they enter the thermal bath soon after they are produced, because the decay temperature is comparable to the Wino/higgsino mass. Their abundance is fixed when the pair annihilation rate becomes smaller than the expansion rate of the Universe. After that, they decoupled from the thermal bath and their number in a comoving volume is fixed. In the case of the Wino-like neutralino LSP, the thermal relic density is given by $[87,88]$

$$
\Omega_{\chi}^{(\text {thermal })} h^{2} \simeq 0.1 \times\left(\frac{m_{\chi}}{2.8 \times 10^{3} \mathrm{GeV}}\right)^{2}
$$

If the LSP is the higgsino, ${ }^{20}$ it is given by $[73,74,87]$

$$
\Omega_{\chi}^{(\text {thermal })} h^{2} \sim 0.1 \times\left(\frac{\mu}{10^{3} \mathrm{GeV}}\right)^{2} .
$$

Thus the dark matter abundance can be explained by the thermal relic of the Wino- or higgsino-like neutralino LSP.

\subsection{AD baryogenesis in LVS model in the geometric regime}

In this subsection, we apply this supergravity model to the AD baryogenesis, assuming that the inflaton is coming from a stack of the visible brane. There are several new ingredients for the AD baryogenesis, such as an enhanced coupling with the inflaton and the dilution

\footnotetext{
${ }^{20}$ If $F^{S} \sim 1 / \mathcal{V}^{2}$ were assumed, one would find this result similarly.
} 
factor $\Delta^{-1}$ by the lightest modulus decay. Note that the other heavy moduli are irrelevant for the present discussion.

We assume that there somehow exists the enhanced coupling with the inflaton in the Kähler potential and a non-renormalizable interaction in the superpotential:

$$
K=c^{2}(\mathcal{V}) \frac{\left|I_{c}\right|^{2}\left|\phi_{c}\right|^{2}}{M_{\mathrm{pl}}^{2}}, \quad W=y \frac{\phi^{n}}{M_{\mathrm{pl}}^{n-3}}, \quad \text { where } \phi_{c} \equiv Z_{\phi}^{1 / 2} \phi .
$$

We would like to emphasize here that, even if $H_{\text {inf }} \lesssim m_{\tau_{1}} \sim m_{0}, m_{0} \ll c H_{\text {inf }}$ is possible due to the large $c$, and the condition (2.6) can be satisfied.

While the lightest modulus starts to oscillate soon after inflation, the AD field will remain away from the origin due to the inflaton coupling. Then, effective mass matrix of $\mathrm{AD}$ field and the normalized lightest modulus is given by

$$
\left(\begin{array}{cc}
c^{2} H^{2} & -\frac{m_{3 / 2}^{2}}{\mathcal{V}} \frac{\left\langle\phi_{c}\right\rangle}{M_{\mathrm{pl}}}+c^{2} H^{2}\left(\frac{\left\langle\phi_{c}\right\rangle}{M_{\mathrm{pl}}}\right) \\
-\frac{m_{3 / 2}^{2}}{\mathcal{V}} \frac{\left\langle\phi_{c}\right\rangle}{M_{\mathrm{pl}}}+c^{2} H^{2}\left(\frac{\left\langle\phi_{c}\right\rangle}{M_{\mathrm{pl}}}\right) & m_{\tau_{1}}^{2}
\end{array}\right)
$$

for $m_{0} / c<H \lesssim H_{\text {inf }}$. (Note that the kinetic term is diagonal up to $\phi_{c} / M_{\mathrm{pl}}$.) Here we could find contribution to the mixing from $\delta \tau_{i} \delta \phi_{c}\left(\partial_{\tau_{i}} m_{0}^{2}\right) \phi_{c} \sim-\left(m_{3 / 2}^{2} \phi_{c} /\left(\mathcal{V} M_{\mathrm{pl}}\right)\right) \delta \phi_{1} \delta \phi_{c}$, $\left\langle\phi_{c}\right\rangle \sim\left(\mathrm{cHM}_{*}^{n-3}\right)^{1 /(n-2)}$ and the inflaton coupling to the modulus is neglected since it will be dependent on the inflation model. As a consequence, for $\left\langle\phi_{c}\right\rangle \lesssim c H M_{\mathrm{pl}} m_{\tau_{1}} \mathcal{V} / m_{3 / 2}^{2} \sim$ $c H M_{\mathrm{pl}} \mathcal{V}^{3 / 2} /\left\langle W_{\text {eff }}\right\rangle$, such a picture is valid until $H \sim m_{0} / c$. Around $H \sim m_{0} / c$, the AD fields also starts oscillating and the effective matrix becomes

$$
\left(\begin{array}{cc}
m_{0}^{2} & -\frac{m_{3 / 2}^{2}}{\mathcal{V}} \frac{\left\langle\phi_{c}\right\rangle}{M_{\mathrm{pl}}} \\
-\frac{m_{3 / 2}^{2}}{\mathcal{V}} \frac{\left\langle\phi_{c}\right\rangle}{M_{\mathrm{pl}}} & m_{\tau_{1}}^{2}
\end{array}\right)
$$

Then, so far as $\phi_{c, \text { osc }} \lesssim M_{\mathrm{pl}} m_{0} m_{\tau_{1}} \mathcal{V} / m_{3 / 2}^{2} \sim M_{\mathrm{pl}}$, the off-diagonal components is smaller than the diagonal ones. Furthermore, with respect to the phase of the MSSM flat direction, the mass mixing between it and the modulus is always smaller than the curvature of the phase direction due to the fact of $\left(\frac{\phi_{c}}{M_{\mathrm{pl}}}\right) \ll 1$. Thus, we conclude that around $H \sim m_{0} / c$ the true vacuum for the $\mathrm{AD}$ fields and the modulus becomes stable; the $\mathrm{AD}$ fields starts oscillating and the baryon asymmetry is generated.

The final baryon asymmetry is written as eq. (2.30), assuming that the lightest modulus dominates over the energy density in the Universe. Since the dilution factor $\Delta^{-1}$ is already computed in the previous subsection, let us consider the typical magnitude of $c$. In the geometric regime there will be heavy KK modes and a massive one in the Landau level on the visible fluxed-brane wrapping on the local 4-cycle $\tau_{2}$. They will be interacting with light modes, and the contact term $K=\left|I_{c}\right|^{2}\left|\phi_{c}\right|^{2} / \tilde{M}^{2}$ will be generated after integrating them out. Thus the mass scale $\tilde{M}$ is expected as

$$
\tilde{M} \sim M_{K K}^{\prime} \sim \frac{M_{\mathrm{pl}}}{\tau_{2}^{1 / 4} \mathcal{V}^{1 / 2}} \rightarrow c \sim \mathcal{V}^{1 / 2}
$$


Note that the condition of eq. (2.10) is not satisfied in this case. Thus the resultant baryon asymmetry is given by

$$
\begin{aligned}
\frac{n_{B}}{s}\left(t_{0}\right) & \sim 10^{-10} \delta_{\text {eff }}\left(\frac{c}{10^{2}}\right)\left(\frac{T_{\mathrm{dec}}^{\phi_{1}}}{10^{3} \mathrm{GeV}}\right)\left(\frac{m_{0}}{10^{7} \mathrm{GeV}}\right)^{-1}\left(\frac{\phi_{c, \mathrm{osc}}}{10^{14} \mathrm{GeV}}\right)^{2}\left(\frac{\Delta \phi_{1}}{M_{\mathrm{pl}}}\right)^{-2} \\
& \sim \delta_{\mathrm{eff}} \frac{\left\langle W_{\mathrm{eff}}\right\rangle}{\log (\mathcal{V})^{1 / 4} \mathcal{V}} \times\left(\frac{\Delta \phi_{1}}{M_{\mathrm{pl}}}\right)^{-2} \sim \delta_{\mathrm{eff}} \frac{1}{\log (\mathcal{V})^{1 / 4}} \frac{m_{3 / 2}}{M_{\mathrm{pl}}} \times\left(\frac{\Delta \phi_{1}}{M_{\mathrm{pl}}}\right)^{-2} .
\end{aligned}
$$

for a numerical example given in the previous subsection. Here we have used the result of $\phi_{c, \text { osc }}$ for $n=6$ in $W \sim y \phi^{n}$. In this case the baryon would be explained for $m_{3 / 2}=$ $\mathcal{O}\left(10^{9}\right) \mathrm{GeV}, \mathcal{V}=\mathcal{O}\left(10^{5}\right)$.

Considering the unification of the cutoff scale, one might study the case of

$$
\tilde{M} \sim M_{*} \sim M_{\mathrm{pl}} / \mathcal{V}^{1 / 3} \rightarrow c \sim \mathcal{V}^{1 / 3},
$$

though this operator is understood in terms of the winding mode. Again, eq. (2.10) is not satisfied. For such a case, one finds

$$
\begin{aligned}
\frac{n_{B}}{s}\left(t_{0}\right) & \sim 10^{-10} \delta_{\text {eff }}\left(\frac{c}{10}\right)\left(\frac{T_{\mathrm{dec}}^{\phi_{1}}}{10^{3} \mathrm{GeV}}\right)\left(\frac{m_{0}}{10^{6} \mathrm{GeV}}\right)^{-1}\left(\frac{\phi_{c, \mathrm{osc}}}{10^{14} \mathrm{GeV}}\right)^{2} \times\left(\frac{\Delta \phi_{1}}{M_{\mathrm{pl}}}\right)^{-2} \\
& \sim \delta_{\mathrm{eff}} \frac{\left\langle W_{\mathrm{eff}}\right\rangle}{\log (\mathcal{V})^{1 / 4} \mathcal{V}^{7 / 6}} \times\left(\frac{\Delta \phi_{1}}{M_{\mathrm{pl}}}\right)^{-2} \sim \delta_{\mathrm{eff}} \frac{1}{\log (\mathcal{V})^{1 / 4} \mathcal{V}^{1 / 6}} \frac{m_{3 / 2}}{M_{\mathrm{pl}}} \times\left(\frac{\Delta \phi_{1}}{M_{\mathrm{pl}}}\right)^{-2} .
\end{aligned}
$$

Thus, in this latter case, the baryon asymmetry will be explained for $m_{3 / 2} \sim 10^{9} \mathrm{GeV}$ and $\Delta \phi_{1} \lesssim M_{\mathrm{pl}}$. Here we have adopted the result for $n=6$, again.

\section{LVS with three Kähler moduli in the singular regime}

Here let us study the local model with visible branes on singularities [66], instead of the geometric regime model. (For model building, see e.g. [89-94].) The branes on the singularity lead to a chiral theory with anomalous $\mathrm{U}(1)$ symmetries. We consider the following Kähler and super-potentials:

$$
\begin{aligned}
K & =-2 \log \left(\tau_{1}^{3 / 2}-\tau_{3}^{3 / 2}+\xi / 2\right)+\frac{\left(\tau_{2}+V_{\mathrm{U}(1)}\right)^{2}}{\tau_{1}^{3 / 2}}+\frac{|\phi|^{2}}{\tau_{1}}, \\
W & =W_{\text {flux }}+e^{-a T_{3}}+W_{\mathrm{MSSM}}(\phi), \\
f_{\mathrm{vis}, a} & =\frac{1}{4 \pi}\left(T_{2}+h_{a} S\right),
\end{aligned}
$$

where $V_{\mathrm{U}(1)}$ denotes the anomalous $\mathrm{U}(1)$ multiplet, $\left|W_{\text {flux }}\right|=\mathcal{O}(1)$, which is general in the flux vacua, and $\tau_{i}=T_{i}+T_{i}^{\dagger}$. Thus, by replacing $W_{\text {eff }}$ with $W_{\text {flux }}$, all the results including soft masses are the same as those of the geometric regime, except for $T_{2}$ and $V_{\mathrm{U}(1)} \cdot T_{2}$ is stabilized via the D-term condition, $D_{\mathrm{U}(1)} \sim \partial_{T_{2}} K \propto \tau_{2}=0$, and it is the Goldstone multiplet absorbed into the $V_{\mathrm{U}(1)}$. Then one finds

$$
m_{\tau_{2}} \sim m_{V_{\mathrm{U}(1)}} \sim M_{\text {string }} \sim \frac{M_{\mathrm{pl}}}{\mathcal{V}^{1 / 2}}, \quad F^{T_{2}} \propto \partial_{T_{2}} K \propto \tau_{2}=0
$$


The result is reasonable since $T_{2}$ describes the volume of the singularity. Thus, in order to realize the SM gauge couplings, $h_{a}\langle S\rangle \simeq 25$ is required, where $h_{a}$ may take slightly different values depending on the gauge group. Note also that the cutoff scale is estimated in a similar fashion to the case of the geometric regime, and it is given by $M_{*} \sim M_{\mathrm{pl}} / \mathcal{V}^{1 / 3} \sim M_{\text {wind }}$.

Hereafter we focus on the following case,

$$
\mathcal{V}=\mathcal{O}\left(10^{7}-10^{8}\right), \quad \frac{F^{S}}{S+S^{\dagger}} \sim \frac{1}{\mathcal{V}^{2}}
$$

in order to obtain the moduli masses and SUSY-breaking soft masses which are similar to the numerical example in the geometric regime. Then one finds

$$
M_{a} \sim \frac{F^{S}}{S+S^{\dagger}} \sim \mathcal{V}^{-2} \sim 10^{2} \mathrm{GeV}-10 \mathrm{TeV}
$$

It is known that, if chiral multiplets charged under the visible gauge group have the $\mathrm{U}(1)$ charge, or if the $\mathrm{U}(1)$ interaction is not extremely weak, there will be 1-loop threshold corrections to the soft masses from the heavy gauge multiplet [95]. This will lead to rather heavy gauginos and tachyonic scalars. In order to avoid this, we neglect those threshold corrections, assuming that the MSSM fields are not charged under the U(1) and that the MSSM singlet fields charged under the $\mathrm{U}(1)$ are heavier than the gravitino via instanton $e^{-2 \pi\left(T_{2}+h S\right)}$.

Let us express the moduli fields in terms of the mass eigenstates as follows [84]:

$$
\begin{gathered}
\left(\begin{array}{c}
\delta \tau_{1} \\
\delta \tau_{2} \\
\delta \tau_{3} \\
\delta\left(1 / g_{s}\right)
\end{array}\right) \sim\left(\begin{array}{c}
\mathcal{V}^{2 / 3} \\
0 \\
\frac{m_{3 / 2}}{m_{\tau_{3}}} \\
\mathcal{V}^{-1 / 2}
\end{array}\right) \delta \phi_{1}+\left(\begin{array}{c}
0 \\
\mathcal{V}^{1 / 2} \\
0 \\
0
\end{array}\right) \delta \phi_{2}+\left(\begin{array}{c}
\mathcal{V}^{1 / 6} \\
0 \\
\mathcal{V}^{1 / 2} \\
\mathcal{V}^{-1}
\end{array}\right) \delta \phi_{3}+\left(\begin{array}{c}
\mathcal{V}^{1 / 6} \\
0 \\
\mathcal{V}^{-1 / 2} \\
\mathcal{O}(1)
\end{array}\right) \delta \phi_{s} \\
\left(\begin{array}{c}
\delta \sigma_{1} \\
\delta \sigma_{2} \\
\delta \sigma_{3} \\
\delta C_{0}^{\mathrm{RR}}
\end{array}\right) \sim\left(\begin{array}{c}
\mathcal{V}^{2 / 3} \\
0 \\
0 \\
0
\end{array}\right) \delta a_{1}+\left(\begin{array}{c}
0 \\
\mathcal{V}^{1 / 2} \\
0 \\
0
\end{array}\right) \delta a_{2}+\left(\begin{array}{c}
\mathcal{V}^{1 / 6} \\
0 \\
\mathcal{V}^{1 / 2} \\
\mathcal{V}^{-1}
\end{array}\right) \delta a_{3}+\left(\begin{array}{c}
0 \\
0 \\
\mathcal{V}^{-1 / 2} \\
\mathcal{O}(1)
\end{array}\right) \delta a_{s}
\end{gathered}
$$

Note that $\delta \tau_{2}$ and $\delta \sigma_{2}$ do not mix with the other fields because of $\tau_{2}=0 . \delta a_{1}$ remains massless as before.

Suppose that the inflation scale is smaller than or comparable to the lightest modulus mass: $H_{\text {inf }} \lesssim m_{\tau_{1}}$. Then the lightest modulus $\delta \phi_{1}$ may induce the cosmological moduli problem, and if so, its decay is relevant for cosmology. The couplings of the lightest modulus to the visible sector are different from those in the geometric regime due to the fact

$$
\partial_{T_{1,3}} F^{T_{2}} \sim \partial_{T_{1,3}} \partial_{T_{2}} K \sim \tau_{2}=0
$$

In particular, the decay into gauginos and higgsino is suppressed as we shall see later. 
Instead, the moduli-dependent Giudice-Masiero term becomes important:

$$
\begin{aligned}
\int d^{4} \theta \frac{H_{u} H_{d}}{\left(T_{1}+T_{1}^{\dagger}\right)} & =-\int d^{4} \theta \frac{\delta T_{1}^{\dagger}}{\left(T_{1}+T_{1}^{\dagger}\right)} \frac{H_{u} H_{d}}{\left(T_{1}+T_{1}^{\dagger}\right)} \\
& \supset-\frac{1}{M_{\mathrm{pl}}} h_{c} h_{c} \partial^{2} \delta \phi_{1}, \\
\left(\delta \tau_{1} \partial_{\tau_{1}}+\delta \tau_{3} \partial_{\tau_{3}}\right)(B \mu) H_{u} H_{d} & \sim \frac{m_{0}^{2}}{M_{\mathrm{pl}}} \delta \phi_{1} h_{c} h_{c},
\end{aligned}
$$

where $h_{c}$ is the canonically normalized (light) Higgs field. The interaction from the $B \mu$ term is most relevant for the decay of $\delta \phi_{1}$. Then the total decay width is given by

$$
\begin{aligned}
\Gamma_{\phi_{1}} \simeq \Gamma\left(\phi_{1} \rightarrow h h\right) & \sim \frac{1}{4 \pi} \frac{m_{0}^{3}}{M_{\mathrm{pl}}^{2}} \frac{m_{0}}{m_{\tau_{1}}} \\
& \simeq \frac{\log (\mathcal{V})^{2}}{4 \pi} \frac{m_{\tau_{1}}^{3}}{M_{\mathrm{pl}}^{2}} \sim \sqrt{\log (\mathcal{V})} \frac{M_{\mathrm{pl}}}{\mathcal{V}^{9 / 2}},
\end{aligned}
$$

if the decay is kinematically allowed.

Let us comment on other decay modes. Noting that the $\mu$-term is given by

$$
\mu \simeq m_{3 / 2}+\frac{F^{T_{1}}}{2 \tau_{1}} \sim \frac{1}{\log (\mathcal{V}) \mathcal{V}^{2}},
$$

the coupling of $\delta \phi_{1}$ to the higgsino is suppressed by the $\mu$-parameter:

$$
\frac{\mu}{M_{\mathrm{pl}}} \delta \phi_{1} \tilde{h}_{c} \tilde{h}_{c}
$$

On the other hand, the coupling of $\delta \phi_{1}$ to gauginos is given by

$$
\frac{M_{1 / 2}}{M_{\mathrm{pl}}} \delta \phi_{1} \lambda_{c} \lambda_{c}
$$

where we have assumed the dependence of $F^{S}$ on $\mathcal{V}$ as $\partial_{\tau_{1}} F^{S} \sim m_{3 / 2} / \mathcal{V}^{2 / 3}$. In addition, although one-loop suppressed, the anomaly mediation contributes to the coupling to gauginos. Thus, the branching fractions of the decay into gauginos and higgsinos are approximately given by $\left(M_{1 / 2} / m_{\tau_{1}}\right)^{2} / \log (\mathcal{V})^{2} \sim \mathcal{V}^{-1} / \log (\mathcal{V})$ and $\left(\mu / m_{\tau_{1}}\right)^{2} / \log (\mathcal{V})^{2} \sim$ $\mathcal{V}^{-1} / \log (\mathcal{V})^{3}$, respectively.

As in the geometric regime model, one can read the branching fraction of $\phi_{1} \rightarrow 2 a_{1}$ through the axion kinetic term:

$$
B\left(\phi_{1} \rightarrow a_{1} a_{1}\right) \simeq\left(\frac{m_{\tau_{1}}}{m_{0}}\right)^{4} \sim \frac{1}{\log (\mathcal{V})^{2}} \sim 3 \times 10^{-3} .
$$

Thus the produced axions from the modulus decay do not give effect on the BBN or CMB again. Note that the decay to gravitinos is kinematically forbidden.

The decay temperature of the lightest modulus is given by

$$
T_{\mathrm{dec}}^{\phi_{1}} \sim 6 \mathrm{GeV} \times\left(\frac{m_{\tau_{1}}}{1.5 \times 10^{6} \mathrm{GeV}}\right)^{3 / 2} .
$$


Thus the dilution factor by modulus decay will be

$$
\Delta^{-1} \simeq \frac{T_{\mathrm{dec}}^{\phi_{1}}}{T_{\mathrm{dom}}^{\phi_{1}}} \sim 2 \times 10^{-6} \times\left(\frac{m_{\tau_{1}}}{1.5 \times 10^{6} \mathrm{GeV}}\right)^{3 / 2}\left(\frac{T_{R}}{10^{7} \mathrm{GeV}}\right)\left(\frac{\Delta \phi_{1}}{M_{\mathrm{pl}}}\right)^{-2} .
$$

Remember that $\Delta \phi_{1} / M_{\mathrm{pl}} \sim H_{\mathrm{inf}}^{2} / m_{\tau_{1}}^{2}$ for $H_{\mathrm{inf}}<m_{\tau_{1}}$. So, for the modulus mass and the reheating temperature shown in the parenthesis, there is no entropy dilution if the Hubble parameter during inflation is a few orders of magnitude lower than the modulus mass.

Now let us consider the LSP abundance. As one can see from (4.6) and (4.15), the higgsino is likely the LSP. For $\mathcal{V} \simeq 10^{7}$, the LSP mass is given by $m_{\chi} \simeq \mu \sim 1 \mathrm{TeV}$, while the decay temperature is about $200 \mathrm{GeV}$. Thus, soon after the modulus decay, the higgsino LSP will be in equilibrium with the ambient plasma. The right dark matter abundance can be explained by the thermal relic of the higgsino LSP with mass of about $1 \mathrm{TeV}[73,74,87]$. For a smaller value of $\mathcal{V}$, the LSP abundance exceeds the dark matter density. On the other hand, for a slightly larger value of $\mathcal{V}$, it is possible to account for the observed dark matter density by the non-thermal LSP production by the modulus decay.

It is possible to make the higgsino heavier by introducing the dilaton $S$ dependence of the Kähler potential as

$$
\int d^{4} \theta \frac{\left|H_{u}+H_{d}^{\dagger}\right|^{2}}{\left(T_{1}+T_{1}^{\dagger}\right)} z(S+\bar{S}) \equiv \int d^{4} \theta Z\left|H_{u}+H_{d}^{\dagger}\right|^{2} .
$$

The $\mu$-term is then given by

$$
\mu \simeq m_{3 / 2}+F^{I} \partial_{I} \log (Z) \sim \frac{F^{S}}{S+S^{\dagger}} \sim M_{a} \sim \frac{1}{\mathcal{V}^{2}}=\mathcal{O}(1) \mathrm{TeV}
$$

for $\mathcal{V}=4.3 \times 10^{7}$. Alternatively, it is possible to make gauginos lighter by considering the case of $F^{S} \sim\left(\log (\mathcal{V}) \mathcal{V}^{2}\right)^{-1}$ as in the geometric regime. In these cases, if the LSP is a certain mixture of the bino and higgsino of mass $\mathcal{O}(100) \mathrm{GeV}$, its thermal relic can account for the dark matter [96]. The latest XENON100 result has placed a stringent constraint on such well-tempered bino-higgsino LSP scenario [97].

Finally let us consider the origin of the effective operator $K=\left|I_{c}\right|^{2}\left|\phi_{c}\right|^{2} / \tilde{M}^{2}$ and then discuss the result of the $\mathrm{AD}$ baryogenesis. On the visible brane in the singular quiver locus, there will be neither KK modes nor Landau level. However, there are stringy vibration modes. ${ }^{21}$ Hence one expects

$$
\tilde{M} \sim M_{\text {string }} \sim \frac{M_{\mathrm{pl}}}{\mathcal{V}^{1 / 2}} \quad \rightarrow \quad c \sim \mathcal{V}^{1 / 2}
$$

This is similar but slightly different situation with respect to the LVS model in the geometric regime. Note that eq. (2.10) is not satisfied in this case. The final baryon asymmetry is

\footnotetext{
${ }^{21}$ Furthermore the anomalous U(1) gauge multiplet would becomes relevant if both the inflaton and AD fields are charged under the anomalous U(1), such an operator is directly generated in this effective theory after integrating out the heavy multiplet as already mentioned in the section 2.3. However, threshold correction to soft masses also would become relevant.
} 
estimated as

$$
\begin{aligned}
\frac{n_{B}}{s}\left(t_{0}\right) & \sim 10^{-10} \delta_{\mathrm{eff}}\left(\frac{c}{10^{3}}\right)\left(\frac{T_{\mathrm{dec}}^{\phi_{1}}}{1 \mathrm{GeV}}\right)\left(\frac{m_{0}}{10^{7} \mathrm{GeV}}\right)^{-1}\left(\frac{\phi_{c, \mathrm{osc}}}{10^{14} \mathrm{GeV}}\right)^{2}\left(\frac{\Delta \phi_{1}}{M_{\mathrm{pl}}}\right)^{-2} \\
& \sim \delta_{\mathrm{eff}} \frac{\log (\mathcal{V})^{1 / 4}}{\mathcal{V}^{3 / 2}} \times\left(\frac{\Delta \phi_{1}}{M_{\mathrm{pl}}}\right)^{-2} \sim \delta_{\mathrm{eff}} \log (\mathcal{V})^{1 / 4} \frac{m_{0}}{M_{\mathrm{pl}}} \times\left(\frac{\Delta \phi_{1}}{M_{\mathrm{pl}}}\right)^{-2} .
\end{aligned}
$$

For $\mathcal{V}=\mathcal{O}\left(10^{7}-10^{8}\right),\left|W_{\text {flux }}\right|=\mathcal{O}(1)$ and $\Delta \phi_{1} \lesssim M_{\mathrm{pl}}$, we obtain the correct baryon asymmetry. Here we have adopted the result for $n=6$ superpotential.

Considering the unification of the cutoff scale again, it is plausible to find the case of $\tilde{M} \sim M_{*} \sim M_{\mathrm{pl}} / \mathcal{V}^{1 / 3} \rightarrow c \sim \mathcal{V}^{1 / 3}$, since the winding mode results in generating such a operator. Again, eq. (2.10) is not satisfied. Then, one obtains

$$
\begin{aligned}
\frac{n_{B}}{s}\left(t_{0}\right) & \sim 10^{-10} \delta_{\mathrm{eff}}\left(\frac{c}{10^{2}}\right)\left(\frac{T_{\mathrm{dec}}^{\phi_{1}}}{1 \mathrm{GeV}}\right)\left(\frac{m_{0}}{10^{7} \mathrm{GeV}}\right)^{-1}\left(\frac{\phi_{c, \mathrm{osc}}}{10^{14} \mathrm{GeV}}\right)^{2}\left(\frac{\Delta \phi_{1}}{M_{\mathrm{pl}}}\right)^{-2} \\
& \sim \delta_{\mathrm{eff}} \frac{\log (\mathcal{V})^{1 / 4}}{\mathcal{V}^{5 / 3}} \times\left(\frac{\Delta \phi_{1}}{M_{\mathrm{pl}}}\right)^{-2} \sim \delta_{\mathrm{eff}} \frac{\log (\mathcal{V})^{1 / 4}}{\mathcal{V}^{1 / 6}} \frac{m_{0}}{M_{\mathrm{pl}}} \times\left(\frac{\Delta \phi_{1}}{M_{\mathrm{pl}}}\right)^{-2} .
\end{aligned}
$$

Thus for $m_{0}=\mathcal{O}\left(10^{7}\right) \mathrm{GeV}$, the right amount of the baryon asymmetry may be obtained naturally for $\Delta \phi_{1} \lesssim M_{\mathrm{pl}}$. Here we have used the result for $n=6$ superpotential, again.

\section{Discussion and conclusions}

So far we have simply assumed an inflation model satisfying the constraint (3.46). One of such low-scale inflation models is the so called new inflation. For instance, we consider the two-field new inflation model on the visible brane. The superpotential is given by

$$
W=X\left(\mu^{2}-\frac{\psi^{n}}{M^{n-3}}\right),
$$

where $\psi$ is the inflaton and $X$ has a non-zero F-term during inflation. The Hubble parameter during inflation is of order $\mu^{2}$. The cut-off scale $M$ is considered to be the winding scale. We assume that the Kähler potential is such that the inflaton mass during inflation is smaller than the Hubble parameter by at least one order of magnitude. The smallness of $\mu$ can be explained if it arises from the gaugino condensation or instantons. Interestingly, it may be possible to suppress the gravitino production from the inflaton decay [98-102] in the LVS. This provides another motivation for the LVS, when the moduli problem is avoided by considering the low-scale inflation.

We have also clarified the cosmological moduli problem in a concrete realization of the moduli stabilization. In particular, we have pointed out that, even if the inflation scale is generically required to be smaller than the modulus mass in order to avoid decompactification and run-away, the modulus starts coherent oscillations after inflation with a suppressed amplitude of order $H_{\text {inf }}^{2} / m_{\tau}^{2}$. This is the case if the inflaton at the potential minimum is heavier than the moduli. The induced moduli abundance is not negligible unless the Hubble parameter during inflation is many orders of magnitude smaller than the modulus mass. Furthermore, since the Hubble parameter is bounded below for the AD mechanism to work, the moduli abundance can be sizable. Thus, the cosmological moduli problem needs to be considered seriously even if a low-scale inflation is assumed. 
The recent discovery of the SM-like Higgs boson suggests the high-scale SUSY breaking at about $10 \mathrm{TeV}$ up to the PeV scale. Such high-scale SUSY has the cosmological advantage of ameliorating the cosmological moduli problem. However, there is the notorious moduli-induced gravitino problem: the moduli fields generically decay into gravitinos at a sizable branching fraction. Unless the gravitino mass is sufficiently heavy, the gravitino decay produces too many LSPs, whose abundance easily exceeds the observed dark matter density. In order to avoid this problem, we have considered a concrete realization of the moduli stabilization in the LVS, in which the modulus decay into gravitinos is kinematically forbidden. We have shown that the cosmological moduli problem is indeed solved without the LSP overproduction.

Another important issue is the baryogenesis. Although the moduli fields decay before the BBN, the pre-existing baryon asymmetry is diluted by the huge entropy produced by the modulus decay. Therefore, it is important to study an efficient baryogenesis mechanism, and we have focused on the AD baryogenesis, taking also account of possible Q-ball formation. We have shown that the Q balls decay sufficiently fast, both because the SUSY breaking scale is relatively high, and because of the mild hierarchy between the scalar mass and the gaugino mass. We have also studied the enhanced coupling between the inflaton and the AD field, which is expected in the LVS. Interestingly, such an enhanced coupling has turned out to increase the resultant baryon asymmetry by many orders of magnitude. Furthermore, the enhanced coupling makes it easy for the AD field to develop a large field value during inflation, which is non-trivial especially if the inflation scale is bounded above.

The discovery of the Higgs boson with mass of about $125 \mathrm{GeV}$, therefore, is shedding light not only on the origin of mass, but also on the beginning of the hot radiation dominated Universe as well as the origin of matter.

\section{Acknowledgments}

TH would like to thank to H. Abe and T. Kobayashi for fruitful discussions on the massive modes in extra dimensions. This work was supported by the Grant-in-Aid for Scientific Research on Innovative Areas (No.24111702, No.21111006 and No.23104008) [FT], Scientific Research (A) (No.22244030 and No.21244033 [FT]), and JSPS Grant-in-Aid for Young Scientists (B) (No.24740135) [FT]. This work was also supported by World Premier International Center Initiative (WPI Program), MEXT, Japan.

\section{A Example of $c$}

\section{A.1 Examples: heavy chiral fields}

Consider the Kähler potential below with a volume modulus $T=\mathcal{V}^{2 / 3}$ and the superpotential eq. (2.35):

$$
\begin{aligned}
K_{\text {moduli }} & =-2 M_{\mathrm{pl}}^{2} \log (\mathcal{V})=-3 M_{\mathrm{pl}}^{2} \log \left(T+T^{\dagger}\right), \\
Z_{H} & =Z_{I}=Z_{\phi}=\frac{1}{\mathcal{V}^{2 / 3}}=\frac{1}{\left(T+T^{\dagger}\right)}
\end{aligned}
$$


In this case, one finds

$$
\tilde{M}=\frac{M_{\mathrm{pl}}}{\mathcal{V}^{1 / 3}} \sim M_{\text {wind }}
$$

This $\tilde{M}$ coincides with the physical heavy mass $M_{H}^{\mathrm{phys}}=e^{K / 2} M_{\mathrm{pl}} / Z$ and also the cutoff scale $M_{*}$ in the physical superpotential

$$
W=y^{\text {phys }} \frac{\phi_{c}^{n}}{M_{*}^{n-3}}=\frac{e^{K / 2} y}{\sqrt{Z^{3}}} \frac{\phi^{n}}{\sqrt{Z^{n-3}} M_{\mathrm{pl}}^{n-3}} .
$$

Note that this is understood as a kind of local models because $y^{\text {phys }}$ does not depend on $\mathcal{V}$.

\section{A.2 Examples: heavy vector field}

Consider the Kähler potential below with two moduli $T=\mathcal{V}^{2 / 3}$ and $T_{v}$ eq. (2.37) and (2.38):

$$
\begin{aligned}
K_{\text {moduli }} & =-2 M_{\mathrm{pl}}^{2} \log (\mathcal{V})+M_{\mathrm{pl}}^{2} \frac{\left(T_{v}+T_{v}+V_{H}\right)^{2}}{\mathcal{V}}, \\
f & =\frac{T_{v}}{4 \pi}+\text { dilaton. }
\end{aligned}
$$

Thus it is easy to read the gauge boson mass in the vanishing Fayet-Iliopoulos term $\propto T_{v}$ limit:

$$
\tilde{M} \sim M_{H} \sim M_{\text {string }} \sim \frac{M_{\mathrm{pl}}}{\mathcal{V}^{1 / 2}} .
$$

Here we assumed that the gauge coupling, which will be given by the dilaton, is of $O(1)$. If one considers the relevant Kähler potential is given by

$$
\begin{aligned}
K_{\text {moduli }} & =-3 M_{\mathrm{pl}}^{2} \log \left(T+T^{\dagger}+V_{H}\right), \\
f & =\frac{T}{4 \pi},
\end{aligned}
$$

the D-term condition $D \sim 1 / T-q_{\psi}\left|\psi_{c}\right|^{2} \sim 0$ becomes important. Then one finds $M_{H}=$ $g \sqrt{\left|K^{\prime}\right|} \sim M_{\mathrm{pl}} / \mathcal{V}^{2 / 3} \sim M_{K K}$ and

$$
\tilde{M} \sim \sqrt{\left|K^{\prime}\right|} \sim \frac{M_{\mathrm{pl}}}{\mathcal{V}^{1 / 3}} \sim M_{\text {wind }} .
$$

On the other hand, when the relevant Kähler potential is given by

$$
\begin{aligned}
K_{\text {moduli }} & =-3 M_{\mathrm{pl}}^{2} \log \left(T+T^{\dagger}+V_{H}\right)+K_{\text {other moduli }}\left(\Phi+\Phi^{\dagger}+V_{H}\right), \\
f & =\frac{1}{4 \pi}\left(T+\sum_{i} \Phi_{i}\right),
\end{aligned}
$$

it will be possible to study the vanishing FI-term, i.e. $\partial_{T} K+\partial_{\Phi^{i}} K=0$. Thus the gauge boson mass will be $M_{H} \sim g \sqrt{K^{\prime \prime}} \sim M_{\mathrm{pl}} / \mathcal{V}$ [103] while

$$
\tilde{M} \sim \sqrt{K^{\prime \prime}} \sim \frac{M_{\mathrm{pl}}}{\mathcal{V}^{2 / 3}} \sim M_{K K} .
$$

Typical values of $c$ will be exhibited in table 2 . 


\begin{tabular}{|c|c|c|c|}
\hline$c^{2}$ & $\mathcal{V}^{2 / 3}$ & $\mathcal{V}$ & $\mathcal{V}^{4 / 3}$ \\
\hline & 10 & 30 & $10^{2}$ \\
& $10^{2}$ & $10^{3}$ & $10^{4}$ \\
& $10^{3}$ & $10^{5}$ & $10^{6}$ \\
& $10^{9}$ & $10^{14}$ & $10^{18}$ \\
\hline
\end{tabular}

Table 2. Varieties of c. $T \equiv \mathcal{V}^{2 / 3}$ is corresponding to the overall volume modulus in supergravity. Note that gauge coupling at the tree level is given by $g^{-2} \sim T / 4 \pi$ on the D7-brane in the bulk.

Open Access. This article is distributed under the terms of the Creative Commons Attribution License which permits any use, distribution and reproduction in any medium, provided the original author(s) and source are credited.

\section{References}

[1] ATLAS and CMS collaborations, talks given at CERN on July 42012.

[2] ATLAS collaboration, G. Aad et al., Combined search for the Standard Model Higgs boson using up to $4.9 \mathrm{fb}^{-1}$ of pp collision data at $\sqrt{\mathrm{s}}=7 \mathrm{TeV}$ with the ATLAS detector at the LHC, Phys. Lett. B 710 (2012) 49 [arXiv:1202.1408] [inSPIRE].

[3] CMS collaboration, S. Chatrchyan et al., Search for the standard model Higgs boson decaying into two photons in pp collisions at $\sqrt{s}=7 \mathrm{TeV}$, Phys. Lett. B 710 (2012) 403 [arXiv: 1202 .1487] [INSPIRE].

[4] J. Elias-Miro, J.R. Espinosa, G.F. Giudice, G. Isidori, A. Riotto and A. Strumia, Higgs mass implications on the stability of the electroweak vacuum, Phys. Lett. B 709 (2012) 222 [arXiv:1112.3022] [INSPIRE].

[5] G. Coughlan, W. Fischler, E.W. Kolb, S. Raby and G.G. Ross, Cosmological Problems for the Polonyi Potential, Phys. Lett. B 131 (1983) 59 [INSPIRE].

[6] J.R. Ellis, D.V. Nanopoulos and M. Quirós, On the Axion, Dilaton, Polonyi, Gravitino and Shadow Matter Problems in Supergravity and Superstring Models, Phys. Lett. B 174 (1986) 176 [INSPIRE].

[7] A. Goncharov, A.D. Linde and M. Vysotsky, Cosmological Problems For Spontaneously Broken Supergravity, Phys. Lett. B 147 (1984) 279 [InSPIRE].

[8] M. Endo, K. Hamaguchi and F. Takahashi, Moduli-induced gravitino problem, Phys. Rev. Lett. 96 (2006) 211301 [hep-ph/0602061] [INSPIRE].

[9] S. Nakamura and M. Yamaguchi, Gravitino production from heavy moduli decay and cosmological moduli problem revived, Phys. Lett. B 638 (2006) 389 [hep-ph/0602081] [INSPIRE].

[10] M. Dine, R. Kitano, A. Morisse and Y. Shirman, Moduli decays and gravitinos, Phys. Rev. D 73 (2006) 123518 [hep-ph/0604140] [inSPIRE].

[11] M. Endo, K. Hamaguchi and F. Takahashi, Moduli/Inflaton Mixing with Supersymmetry Breaking Field, Phys. Rev. D 74 (2006) 023531 [hep-ph/0605091] [INSPIRE].

[12] M. Fukugita and T. Yanagida, Baryogenesis Without Grand Unification, Phys. Lett. B 174 (1986) 45 [INSPIRE]. 
[13] I. Affleck and M. Dine, A New Mechanism for Baryogenesis, Nucl. Phys. B 249 (1985) 361 [INSPIRE].

[14] S.R. Coleman, Q Balls, Nucl. Phys. B 262 (1985) 263 [Erratum ibid. B 269 (1986) 744] [INSPIRE].

[15] A. Kusenko, Solitons in the supersymmetric extensions of the standard model, Phys. Lett. B 405 (1997) 108 [hep-ph/9704273] [INSPIRE].

[16] A. Kusenko and M.E. Shaposhnikov, Supersymmetric Q balls as dark matter, Phys. Lett. B 418 (1998) 46 [hep-ph/9709492] [INSPIRE].

[17] V. Balasubramanian, P. Berglund, J.P. Conlon and F. Quevedo, Systematics of moduli stabilisation in Calabi-Yau flux compactifications, JHEP 03 (2005) 007 [hep-th/0502058] [INSPIRE].

[18] M. Dine, L. Randall and S.D. Thomas, Supersymmetry breaking in the early universe, Phys. Rev. Lett. 75 (1995) 398 [hep-ph/9503303] [INSPIRE].

[19] M. Dine, L. Randall and S.D. Thomas, Baryogenesis from flat directions of the supersymmetric standard model, Nucl. Phys. B 458 (1996) 291 [hep-ph/9507453] [INSPIRE].

[20] T. Gherghetta, C.F. Kolda and S.P. Martin, Flat directions in the scalar potential of the supersymmetric standard model, Nucl. Phys. B 468 (1996) 37 [hep-ph/9510370] [InSPIRE].

[21] A. Basboll, A Complete and minimal catalogue of MSSM gauge invariant monomials, Int. J. Mod. Phys. A 25 (2010) 3375 [arXiv:0910.0244] [INSPIRE].

[22] K. Enqvist and J. McDonald, Q balls and baryogenesis in the MSSM, Phys. Lett. B 425 (1998) 309 [hep-ph/9711514] [INSPIRE].

[23] K. Enqvist and J. McDonald, B-ball baryogenesis and the baryon to dark matter ratio, Nucl. Phys. B 538 (1999) 321 [hep-ph/9803380] [INSPIRE].

[24] S. Kasuya and M. Kawasaki, Q-Ball formation in the gravity mediated SUSY breaking scenario, Phys. Rev. D 62 (2000) 023512 [hep-ph/0002285] [INSPIRE].

[25] S. Kasuya and M. Kawasaki, Q-ball formation: Obstacle to Affleck-Dine baryogenesis in the gauge mediated SUSY breaking?, Phys. Rev. D 64 (2001) 123515 [hep-ph/0106119] [INSPIRE].

[26] A.G. Cohen, S.R. Coleman, H. Georgi and A. Manohar, The Evaporation Of Q Balls, Nucl. Phys. B 272 (1986) 301 [inSPIRE].

[27] B. Dutta and K. Sinha, Affleck-Dine Baryogenesis in Effective Supergravity, Phys. Rev. D 82 (2010) 095003 [arXiv: 1008.0148] [INSPIRE].

[28] D. Marsh, Towards Constraining Affleck-Dine Baryogenesis, JHEP 05 (2012) 041 [arXiv: 1108.4687] [INSPIRE].

[29] B. Dutta and K. Sinha, Holomorphic Bisectional Curvatures, Supersymmetry Breaking and Affleck-Dine Baryogenesis, arXiv:1205.6267 [INSPIRE].

[30] J.P. Conlon, R. Kallosh, A.D. Linde and F. Quevedo, Volume Modulus Inflation and the Gravitino Mass Problem, JCAP 09 (2008) 011 [arXiv:0806.0809] [INSPIRE].

[31] S. Kachru, R. Kallosh, A.D. Linde, J.M. Maldacena, L.P. McAllister, and S.P. Trivedi, Towards inflation in string theory, JCAP 10 (2003) 013 [hep-th/0308055] [INSPIRE].

[32] K. Choi and K.S. Jeong, Supersymmetry breaking and moduli stabilization with anomalous U(1) gauge symmetry, JHEP 08 (2006) 007 [hep-th/0605108] [INSPIRE]. 
[33] F. Brummer, A. Hebecker and M. Trapletti, SUSY breaking mediation by throat fields, Nucl. Phys. B 755 (2006) 186 [hep-th/0605232] [INSPIRE].

[34] H. Abe and Y. Sakamura, Roles of Z(2)-odd $N=1$ multiplets in off-shell dimensional reduction of $5 D$ supergravity, Phys. Rev. D 75 (2007) 025018 [hep-th/0610234] [INSPIRE].

[35] H. Abe, T. Higaki, T. Kobayashi and Y. Omura, Dynamically sequestered F-term uplifting in extra dimension, JHEP 04 (2008) 072 [arXiv: 0801.0998] [INSPIRE].

[36] P.G. Camara, E. Dudas and E. Palti, Massive wavefunctions, proton decay and FCNCs in local F-theory GUTs, JHEP 12 (2011) 112 [arXiv:1110.2206] [INSPIRE].

[37] E. Dudas and G. von Gersdorff, Universal contributions to scalar masses from five dimensional supergravity, arXiv: 1207.0815 [INSPIRE].

[38] J.P. Conlon and E. Palti, On Gauge Threshold Corrections for Local IIB/F-theory GUTs, Phys. Rev. D 80 (2009) 106004 [arXiv:0907.1362] [INSPIRE].

[39] J.P. Conlon, Gauge Threshold Corrections for Local String Models, JHEP 04 (2009) 059 [arXiv:0901.4350] [INSPIRE].

[40] J.P. Conlon and E. Palti, Gauge Threshold Corrections for Local Orientifolds, JHEP 09 (2009) 019 [arXiv:0906.1920] [INSPIRE].

[41] J.P. Conlon, M. Goodsell and E. Palti, One-loop Yukawa Couplings in Local Models, JHEP 11 (2010) 087 [arXiv:1007.5145] [INSPIRE].

[42] I. Antoniadis, C. Bachas and E. Dudas, Gauge couplings in four-dimensional type-I string orbifolds, Nucl. Phys. B 560 (1999) 93 [hep-th/9906039] [INSPIRE].

[43] S. Kachru, R. Kallosh, A.D. Linde and S.P. Trivedi, De Sitter vacua in string theory, Phys. Rev. D 68 (2003) 046005 [hep-th/0301240] [INSPIRE].

[44] R. Blumenhagen, B. Körs, D. Lüst and S. Stieberger, Four-dimensional String Compactifications with D-branes, Orientifolds and Fluxes, Phys. Rept. 445 (2007) 1 [hep-th/0610327] [INSPIRE].

[45] R. Blumenhagen, S. Moster and E. Plauschinn, String GUT Scenarios with Stabilised Moduli, Phys. Rev. D 78 (2008) 066008 [arXiv:0806.2667] [InSPIRE].

[46] J.P. Conlon, F. Quevedo and K. Suruliz, Large-volume flux compactifications: Moduli spectrum and D3/D7 soft supersymmetry breaking, JHEP 08 (2005) 007 [hep-th/0505076] [INSPIRE].

[47] M. Cicoli, J.P. Conlon and F. Quevedo, General Analysis of LARGE Volume Scenarios with String Loop Moduli Stabilisation, JHEP 10 (2008) 105 [arXiv:0805.1029] [INSPIRE].

[48] M. Cicoli, A. Maharana, F. Quevedo and C. Burgess, De Sitter String Vacua from Dilaton-dependent Non-perturbative Effects, JHEP 06 (2012) 011 [arXiv:1203.1750] [INSPIRE].

[49] R. Blumenhagen and M. Schmidt-Sommerfeld, Power Towers of String Instantons for $N=1$ Vacua, JHEP 07 (2008) 027 [arXiv: 0803.1562] [INSPIRE].

[50] R. Blumenhagen, X. Gao, T. Rahn and P. Shukla, A Note on Poly-Instanton Effects in Type IIB Orientifolds on Calabi-Yau Threefolds, JHEP 06 (2012) 162 [arXiv:1205.2485] [INSPIRE]. 
[51] M. Cicoli, C. Burgess and F. Quevedo, Anisotropic Modulus Stabilisation: Strings at LHC Scales with Micron-sized Extra Dimensions, JHEP 10 (2011) 119 [arXiv:1105.2107] [INSPIRE].

[52] M. Cicoli, F.G. Pedro and G. Tasinato, Poly-instanton Inflation, JCAP 12 (2011) 022 [arXiv:1110.6182] [INSPIRE].

[53] M. Cicoli, F.G. Pedro and G. Tasinato, Natural Quintessence in String Theory, JCAP 07 (2012) 044 [arXiv: 1203.6655] [INSPIRE].

[54] M. Cicoli, M. Goodsell and A. Ringwald, The type IIB string axiverse and its low-energy phenomenology, arXiv:1206.0819 [INSPIRE].

[55] O. DeWolfe, A. Giryavets, S. Kachru and W. Taylor, Enumerating flux vacua with enhanced symmetries, JHEP 02 (2005) 037 [hep-th/0411061] [INSPIRE].

[56] T. Higaki and T. Kobayashi, Note on moduli stabilization, supersymmetry breaking and axiverse, Phys. Rev. D 84 (2011) 045021 [arXiv: 1106.1293] [InSPIRE].

[57] K. Choi, A. Falkowski, H.P. Nilles and M. Olechowski, Soft supersymmetry breaking in KKLT flux compactification, Nucl. Phys. B 718 (2005) 113 [hep-th/0503216] [INSPIRE].

[58] A. Saltman and E. Silverstein, The scaling of the no scale potential and de Sitter model building, JHEP 11 (2004) 066 [hep-th/0402135] [INSPIRE].

[59] O. Lebedev, H.P. Nilles and M. Ratz, De Sitter vacua from matter superpotentials, Phys. Lett. B 636 (2006) 126 [hep-th/0603047] [INSPIRE].

[60] E. Dudas, C. Papineau and S. Pokorski, Moduli stabilization and uplifting with dynamically generated F-terms, JHEP 02 (2007) 028 [hep-th/0610297] [INSPIRE].

[61] H. Abe, T. Higaki, T. Kobayashi and Y. Omura, Moduli stabilization, F-term uplifting and soft supersymmetry breaking terms, Phys. Rev. D 75 (2007) 025019 [hep-th/0611024] [INSPIRE].

[62] R. Kallosh and A.D. Linde, O'kklt, JHEP 02 (2007) 002 [hep-th/0611183] [INSPIRE].

[63] H. Abe, T. Higaki and T. Kobayashi, More about F-term uplifting, Phys. Rev. D 76 (2007) 105003 [arXiv:0707.2671] [INSPIRE].

[64] I. Bena, M. Graña, S. Kuperstein and S. Massai, Anti-D3's - Singular to the Bitter End, arXiv:1206.6369 [INSPIRE].

[65] H. Abe, T. Higaki and T. Kobayashi, Remark on integrating out heavy moduli in flux compactification, Phys. Rev. D 74 (2006) 045012 [hep-th/0606095] [INSPIRE].

[66] R. Blumenhagen, J. Conlon, S. Krippendorf, S. Moster and F. Quevedo, SUSY Breaking in Local String/F-Theory Models, JHEP 09 (2009) 007 [arXiv:0906.3297] [INSPIRE].

[67] J.P. Conlon, D. Cremades and F. Quevedo, Kähler potentials of chiral matter fields for Calabi-Yau string compactifications, JHEP 01 (2007) 022 [hep-th/0609180] [INSPIRE].

[68] J.P. Conlon, S.S. AbdusSalam, F. Quevedo and K. Suruliz, Soft SUSY Breaking Terms for Chiral Matter in IIB String Compactifications, JHEP 01 (2007) 032 [hep-th/0610129] [INSPIRE].

[69] R. Blumenhagen, Gauge Coupling Unification in F-theory Grand Unified Theories, Phys. Rev. Lett. 102 (2009) 071601 [arXiv:0812.0248] [INSPIRE]. 
[70] K. Choi, H.P. Nilles, C.S. Shin and M. Trapletti, Sparticle Spectrum of Large Volume Compactification, JHEP 02 (2011) 047 [arXiv: 1011.0999] [INSPIRE].

[71] J.P. Conlon and F.G. Pedro, Moduli Redefinitions and Moduli Stabilisation, JHEP 06 (2010) 082 [arXiv: 1003.0388] [INSPIRE].

[72] A. Brignole, L.E. Ibáñez and C. Muñoz, Soft supersymmetry breaking terms from supergravity and superstring models, hep-ph/9707209 [INSPIRE].

[73] N. Arkani-Hamed and S. Dimopoulos, Supersymmetric unification without low energy supersymmetry and signatures for fine-tuning at the LHC, JHEP 06 (2005) 073 [hep-th/0405159] [INSPIRE].

[74] G. Giudice and A. Romanino, Split supersymmetry, Nucl. Phys. B 699 (2004) 65 [Erratum ibid. B 706 (2005) 65-89] [hep-ph/0406088] [INSPIRE].

[75] H. Abe, T. Higaki and T. Kobayashi, KKLT type models with moduli-mixing superpotential, Phys. Rev. D 73 (2006) 046005 [hep-th/0511160] [INSPIRE].

[76] M. Haack, D. Krefl, D. Lüst, A. Van Proeyen and M. Zagermann, Gaugino Condensates and D-terms from D7-branes, JHEP 01 (2007) 078 [hep-th/0609211] [INSPIRE].

[77] M. Ibe and T.T. Yanagida, The Lightest Higgs Boson Mass in Pure Gravity Mediation Model, Phys. Lett. B 709 (2012) 374 [arXiv:1112.2462] [INSPIRE].

[78] A. Hebecker, A.K. Knochel and T. Weigand, A Shift Symmetry in the Higgs Sector: Experimental Hints and Stringy Realizations, JHEP 06 (2012) 093 [arXiv:1204.2551] [INSPIRE].

[79] R. Kallosh and A.D. Linde, Landscape, the scale of SUSY breaking and inflation, JHEP 12 (2004) 004 [hep-th/0411011] [INSPIRE].

[80] M. Badziak and M. Olechowski, Volume modulus inflation and a low scale of SUSY breaking, JCAP 07 (2008) 021 [arXiv:0802.1014] [INSPIRE].

[81] M. Badziak and M. Olechowski, Volume modulus inflection point inflation and the gravitino mass problem, JCAP 02 (2009) 010 [arXiv:0810.4251] [INSPIRE].

[82] K. Nakayama, F. Takahashi and T.T. Yanagida, On the Adiabatic Solution to the Polonyi/Moduli Problem, Phys. Rev. D 84 (2011) 123523 [arXiv:1109.2073] [INSPIRE].

[83] J.P. Conlon and F. Quevedo, Astrophysical and cosmological implications of large volume string compactifications, JCAP 08 (2007) 019 [arXiv:0705.3460] [INSPIRE].

[84] M. Cicoli and A. Mazumdar, Reheating for Closed String Inflation, JCAP 09 (2010) 025 [arXiv: 1005.5076] [INSPIRE].

[85] K. Ichikawa, M. Kawasaki, K. Nakayama, M. Senami and F. Takahashi, Increasing effective number of neutrinos by decaying particles, JCAP 05 (2007) 008 [hep-ph/0703034] [INSPIRE].

[86] K.S. Jeong and F. Takahashi, Light Higgsino from Axion Dark Radiation, JHEP 08 (2012) 017 [arXiv:1201.4816] [INSPIRE].

[87] J. Hisano, S. Matsumoto, M. Nagai, O. Saito and M. Senami, Non-perturbative effect on thermal relic abundance of dark matter, Phys. Lett. B 646 (2007) 34 [hep-ph/0610249] [INSPIRE].

[88] G.F. Giudice, M.A. Luty, H. Murayama and R. Rattazzi, Gaugino mass without singlets, JHEP 12 (1998) 027 [hep-ph/9810442] [INSPIRE]. 
[89] G. Aldazabal, L.E. Ibáñez, F. Quevedo and A. Uranga, D-branes at singularities: A Bottom up approach to the string embedding of the standard model, JHEP 08 (2000) 002 [hep-th/0005067] [INSPIRE].

[90] J. Cascales, M. Garcia del Moral, F. Quevedo and A. Uranga, Realistic D-brane models on warped throats: Fluxes, hierarchies and moduli stabilization, JHEP 02 (2004) 031 [hep-th/0312051] [INSPIRE].

[91] J.P. Conlon, A. Maharana and F. Quevedo, Towards Realistic String Vacua, JHEP 05 (2009) 109 [arXiv:0810.5660] [INSPIRE].

[92] S. Krippendorf, M.J. Dolan, A. Maharana and F. Quevedo, D-branes at Toric Singularities: Model Building, Yukawa Couplings and Flavour Physics, JHEP 06 (2010) 092 [arXiv: 1002.1790] [INSPIRE].

[93] M.J. Dolan, S. Krippendorf and F. Quevedo, Towards a Systematic Construction of Realistic D-brane Models on a del Pezzo Singularity, JHEP 10 (2011) 024 [arXiv:1106.6039] [INSPIRE].

[94] M. Cicoli, S. Krippendorf, C. Mayrhofer, F. Quevedo and R. Valandro, D-Branes at del Pezzo Singularities: Global Embedding and Moduli Stabilisation, arXiv:1206.5237 [INSPIRE].

[95] C.S. Shin, Anomalous U(1) Mediation in Large Volume Compactification, JHEP 01 (2012) 084 [arXiv: 1108.5740] [INSPIRE].

[96] N. Arkani-Hamed, A. Delgado and G. Giudice, The well-tempered neutralino, Nucl. Phys. B 741 (2006) 108 [hep-ph/0601041] [INSPIRE].

[97] M. Farina, M. Kadastik, D. Pappadopulo, J. Pata, M. Raidal and A. Strumia, Implications of XENON100 and LHC results for Dark Matter models, Nucl. Phys. B 853 (2011) 607 [arXiv: 1104.3572] [INSPIRE].

[98] M. Kawasaki, F. Takahashi and T. Yanagida, Gravitino overproduction in inflaton decay, Phys. Lett. B 638 (2006) 8 [hep-ph/0603265] [INSPIRE].

[99] M. Kawasaki, F. Takahashi and T. Yanagida, The gravitino-overproduction problem in inflationary universe, Phys. Rev. D 74 (2006) 043519 [hep-ph/0605297] [INSPIRE].

[100] T. Asaka, S. Nakamura and M. Yamaguchi, Gravitinos from heavy scalar decay, Phys. Rev. D 74 (2006) 023520 [hep-ph/0604132] [INSPIRE].

[101] M. Endo, F. Takahashi and T. Yanagida, Anomaly-induced inflaton decay and gravitino-overproduction problem, Phys. Lett. B 658 (2008) 236 [hep-ph/0701042] [INSPIRE].

[102] M. Endo, F. Takahashi and T. Yanagida, Inflaton Decay in Supergravity, Phys. Rev. D 76 (2007) 083509 [arXiv:0706.0986] [INSPIRE].

[103] M. Cicoli, M. Goodsell, J. Jaeckel and A. Ringwald, Testing String Vacua in the Lab: From a Hidden CMB to Dark Forces in Flux Compactifications, JHEP 07 (2011) 114 [arXiv:1103.3705] [INSPIRE]. 